\title{
Isotopy for extended affine Lie algebras and Lie tori
}

\author{
Bruce Allison $^{1 *}$ and John Faulkner ${ }^{2}$ \\ 1 Department of Mathematics and Statistics, University of Victoria, Victoria BC \\ V8W 3P4 Canada ballison@uvic.ca \\ 2 Department of Mathematics, University of Virginia, Charlottesville VA \\ 22904-4137 USA jrf@virginia.edu
}

\begin{abstract}
Summary. Centreless Lie tori have been used by E. Neher to construct all extended affine Lie algebras (EALAs). In this article, we study isotopy for centreless Lie tori, and show that Neher's construction provides a 1-1 correspondence between centreless Lie tori up to isotopy and families of EALAs up to isomorphism. Also, centreless Lie tori can be coordinatized by unital algebras that are in general nonassociative, and, for many types of centreless Lie tori, there are classical definitions of isotopy for the coordinate algebras. We show for those types that an isotope of a Lie torus is coordinatized by an isotope of its coordinate algebra, thereby connecting the two notions of isotopy. In writing the article, we have not assumed prior knowledge of the theories of EALAs, Lie tori or isotopy.
\end{abstract}

Key words: isotope, extended affine Lie algebra, Lie torus, nonassociative algebra, Jordan algebra, alternative algebra.

AMS subject classification: Primary 17B65, 17A01. Secondary 17B60, 17B70, 17C99, $17 \mathrm{D} 05$.

\section{Introduction}

If $A$ is a unital associative algebra and $u$ is invertible in $A$, one can define an algebra $A^{(u)}$, called the $u$-isotope of $A$, which is equal to $A$ as a vector space but has a new product $x \cdot u y=x u y$. This isotope $A^{(u)}$ is again unital and associative but with a shifted identity element $u^{-1}$. More generally there are definitions of isotope for several other classes of unital nonassociative algebras, notably Jordan algebras [Mc2, §I.3.2], alternative algebras [Mc1] and associative algebras with involution [Mc2, §I.3.4]. In each case, the isotope is obtained very roughly by shifting the identity element in the algebra, and two algebras are said to be isotopic if one is isomorphic to an isotope of the other. In the associative case, the $u$-isotope $A^{(u)}$ is isomorphic to $A$ under left multiplication by $u$, and therefore isotopy has not played

\footnotetext{
* Bruce Allison gratefully acknowledges the support of the Natural Sciences and Engineering Research Council of Canada.
} 
a role in associative theory. That is not true in general though, and in particular isotopy plays an important role in Jordan theory (see for example [Mc2, §II.7]).

In contrast, isotopes and isotopy have not been defined for Lie algebras, for the evident reason that Lie algebras are not unital. ${ }^{3}$ In this article we study notions of isotope and isotopy, which were recently introduced in [ABFP2] for a class of graded Lie algebras called Lie tori. The point to emphasize here is that forming an isotope of a Lie torus does not change the multiplication at all, but rather it shifts the grading.

We are primarily interested in the case when the Lie torus is centreless, and there are two basic reasons why we are interested in isotopy in this case. First, centreless Lie tori arise naturally in the construction of families of extended affine Lie algebras (EALAs), and we see in this article that isotopes and isotopy play a natural and fundamental role in the theory of both of these classes of Lie algebras. In fact, we show in section 6 using a construction of E. Neher, that there is a 1-1 correspondence between centreless Lie tori up to isotopy and families of EALAs up to bijective isomorphism. (This terminology is explained in section 6.)

Second, any Lie torus is by definition graded by the group $Q \times \Lambda$, where $Q$ is the root lattice of a finite irreducible root system $\Delta$ and $\Lambda$ is (in this article) a finitely generated free abelian group. Depending on the type of $\Delta$, centreless Lie tori are coordinatized by unital algebras that are in general nonassociative. For example, in types $\mathrm{A}_{1}, \mathrm{~A}_{2}, \mathrm{~A}_{r}(r \geq 3)$ and $\mathrm{C}_{r}(r \geq 4)$ the coordinate algebras are respectively Jordan algebras, alternative algebras, associative algebras and associative algebras with involution. It turns out that an isotope of a centreless Lie torus is coordinatized by an isotope of its coordinate algebra, thereby connecting our notion of isotopy for Lie tori with the classical notions for unital nonassociative algebras. This fact can then be used to obtain necessary and sufficient conditions for two centreless Lie tori of the same type to be isotopic (in terms of their coordinate algebras). We describe these results in detail for types $\mathrm{A}_{1}, \mathrm{~A}_{2}, \mathrm{~A}_{r}(r \geq 3)$ and $\mathrm{C}_{r}(r \geq 4)$ in sections 8, 9, 10 , and 11, and we remark very briefly on the other types in section 12 .

Section 12 also includes a brief discussion of an alternate approach, using multiloops, to constructing centreless Lie tori and studying isotopy.

In writing this article, we have not assumed prior knowledge of the theories of EALAs, Lie tori or isotopy. In fact, we hope that this article will serve as a useful introduction to these theories and their interconnections. For this reason, we begin in sections 2, 3, 4 and 5 by recalling some of the important definitions and facts about EALAs, Lie tori and isotopy for Lie tori. For the same reason, we have included a brief discussion of coordinatization in section 7; and in sections 8 and 9 we have included some arguments that are familiar to experts in Jordan and alternative algebra theory, but may be less familiar to others.

Acknowledgements: We thank Stephen Berman, Erhard Neher and Arturo Pianzola for stimulating conversations about the topics of this paper. We also thank Erhard Neher for his helpful comments about a preliminary version of this article.

Assumptions and notation: Throughout this work we assume that $\mathbb{F}$ is a field of characteristic 0 . All algebras are assumed to be algebras over $\mathbb{F}$, and all algebras (except Lie algebras) are assumed to be unital. We also assume that $\Lambda$ is a finitely

\footnotetext{
${ }^{3}$ There is a notion of isotopy that makes sense for nonunital algebras and hence Lie algebras $[\mathrm{A}]$, but it is quite different from the one discussed in this article.
} 
generated free abelian group. We denote the rank of $\Lambda$, which is an integer $\geq 0$, by $\operatorname{rank}(\Lambda)$.

If $E$ is a Lie algebra and $H$ is an ad-diagonalizable subalgebra of $E$, we let $E_{\rho}=\{x \in E \mid[h, x]=\rho(h) x$ for $h \in H\}$ for $\rho$ in the dual space $H^{*}$ of $H$. Then, we define $\Delta(E, H)=\left\{\rho \in H^{*} \mid E_{\rho} \neq 0\right\}$ and we call elements of $\Delta(E, H)$ roots of $E$ relative to $H$; so in particular, 0 is a root (if $E \neq 0$ ).

Some terminology for graded algebras: If $A=\oplus_{\lambda \in \Lambda} A^{\lambda}$ is a $\Lambda$-graded algebra, the $\Lambda$-support of $A$ is $\operatorname{supp}_{\Lambda}(A)=\left\{\lambda \in \Lambda \mid A^{\lambda} \neq 0\right\}$. If $\Gamma$ is a subgroup of $\Lambda$ and $A$ is a $\Gamma$-graded algebra or vector space, we regard $A$ as $\Lambda$-graded by setting $A^{\lambda}=0$ for $\lambda \in \Lambda \backslash \Gamma$. If $A$ is a $\Lambda$-graded algebra and $A^{\prime}$ is a $\Lambda^{\prime}$-graded algebra, we say that $A$ and $A^{\prime}$ are isograded-isomorphic, written $A \simeq_{i g} A^{\prime}$, if there exists an algebra isomorphism $\eta: A \rightarrow A^{\prime}$ and a group isomorphism $\eta_{\mathrm{gr}}: \Lambda \rightarrow \Lambda^{\prime}$ such that $\eta\left(A^{\lambda}\right)=$ $A^{\prime \eta_{\mathrm{gr}}(\lambda)}$ for $\lambda \in \Lambda$; in that case $\eta_{\mathrm{gr}}$ is uniquely determined by $\eta$ if $\left\langle\operatorname{supp}_{\Lambda}(A)\right\rangle=\Lambda$. $\left(\right.$ Here $\left\langle\operatorname{supp}_{\Lambda}(A)\right\rangle$ denotes the subgroup of $\Lambda$ generated by $\operatorname{supp}_{\Lambda}(A)$.) If $A$ and $A^{\prime}$ are $\Lambda$-graded algebras, we say that $A$ and $A^{\prime}$ are graded-isomorphic, written $A \simeq_{\Lambda} A^{\prime}$, if there is an algebra isomorphism $\eta: A \rightarrow A^{\prime}$ that preserves the grading (that is $\eta_{\mathrm{gr}}=\mathrm{id}$ ). A $\Lambda$-graded algebra with involution is a pair $(A, \iota)$, where $A$ is a $\Lambda$-graded algebra and $\iota$ is an involution (antiautomorphism of period 2) of $A$ that is graded. There is an evident extension of the terms isograded-isomorphic and graded-isomorphic and the notations $\simeq_{i g}$ and $\simeq_{\Lambda}$ for graded algebras with involution. Finally, the group algebra $\mathbb{F}[\Lambda]=\oplus_{\lambda \in \Lambda} \mathbb{F} t^{\lambda}$ of $\Lambda$ with $t^{\lambda} t^{\lambda^{\prime}}=t^{\lambda+\lambda^{\prime}}$ is naturally a $\Lambda$-graded algebra. If we choose a basis $\left\{\lambda_{1}, \ldots, \lambda_{n}\right\}$ for $\Lambda$, then $\mathbb{F}[\Lambda]$ is isograded-isomorphic to the algebra $\mathbb{F}\left[t_{1}^{ \pm 1}, \ldots, t_{n}^{ \pm 1}\right]$ of Laurent polynomials with its natural $\mathbb{Z}^{n}$-grading.

\section{Extended affine Lie algebras}

The definition of an EALA has evolved from [HT], where these algebras were introduced under the name elliptic irreducible quasi-simple Lie algebra, and from [BGK] and $[\mathrm{AABGP}]$, where many of their properties were developed. We will use the definition given in [N2], which has the important advantage that it makes sense over any field of characteristic 0. Some of the facts about EALAs mentioned below were proved in the setting of [AABGP], but they can be verified in a similar fashion in our more general setting.

Recall that an extended affine Lie algebra (EALA) is a triple $(E,(\mid), H)$, where $E$ is a Lie algebra over $\mathbb{F},(\mid)$ is a nondegenerate invariant symmetric bilinear form on $E$, and $H$ is a finite dimensional nonzero self-centralizing ad-diagonalizable subalgebra of $E$, such that a list of axioms labeled as (EA3)(EA6) are satisfied [N2]. For the purposes of this article we do not need the precise statement of these axioms, except to say that they are modeled after the properties of finite dimensional split simple Lie algebras and affine Kac-Moody Lie algebras. If $(E,(\mid), H)$ is an EALA, we also say that $E$ is an $E A L A$ with respect to $(\mid)$ and $H$, or simply that $E$ is an $E A L A$. Roots of $E$ relative to $H$ are simply called roots of $E$.

If $E$ is an EALA, we can as usual transfer the restriction of $(\mid)$ to $H$ to a nondegenerate form $(\mid)$ on the dual space $H^{*}$. If $R=\Delta(E, H), V=\operatorname{span}_{\mathbb{F}}(R)$, and $\operatorname{rad}(V)$ is the radical of the restriction of $(\mid)$ to $V$, then the image of $R$ in 
$V / \operatorname{rad}(V)$ is a finite irreducible root system (including 0) whose type is called the type of $E$. (See the beginning of $\S 3$ below for our conventions about finite irreducible root systems.)

A root $\rho$ of an EALA $E$ is called isotropic if $(\rho, \rho)=0$, and otherwise called nonisotropic. According to one of the axioms for an EALA, the group generated by the isotropic roots is a free abelian group of finite rank, and its rank is called the nullity of $E$. Then the extended affine Lie algebras of nullity 0 and 1 are precisely the finite dimensional split simple Lie algebras and the affine Kac-Moody Lie algebras respectively. (See [ABFP2, Remark 1.2.4] for nullity 0 and [ABGP] for nullity 1.)

If $(E,(\mid), H)$ is an EALA, then so is $(E, a(\mid), H)$ for $a \in \mathbb{F}^{\times}$, and it is sometimes convenient to adjust the form in this way. For this reason, the following is a natural notion of isomorphism for EALAs.

Definition 2.1. If $(E,(\mid), H)$ and $\left(E^{\prime},(\mid)^{\prime}, H^{\prime}\right)$ are $E A L A s$, an isomorphism from $(E,(\mid), H)$ onto $\left(E^{\prime},(\mid)^{\prime}, H^{\prime}\right)$ is a Lie algebra isomorphism $\chi: E \rightarrow E^{\prime}$ such that $(\chi(x) \mid \chi(y))^{\prime}=a(x \mid y)$ for some $a \in \mathbb{F}^{\times}$and all $x, y \in E$, and $\chi(H)=H^{\prime}$. If such a map exists we say that $(E,(\mid), H)$ and $\left(E^{\prime},(\mid)^{\prime}, H^{\prime}\right)$ are isomorphic. For short we often say that $\chi$ is an EALA isomorphism from $E$ onto $E^{\prime}$ and that $E$ and $E^{\prime}$ are isomorphic as EALAs.

Definition 2.2. If $E$ is an $E A L A$, the core of $E$ is the subalgebra $E_{c}$ of $E$ that is generated by the root spaces of $E$ corresponding to nonisotropic roots, and the centreless core of $E$ is $E_{c c}:=E_{c} / Z\left(E_{c}\right){ }^{4}$

\section{Lie tori}

With the construction of families of EALAs in mind, Yoshii gave a definition of a Lie torus in [Y6]. An equivalent definition, which we recall next, was given by Neher in $[\mathrm{N} 1]$.

It will be convenient for us to work with root systems that contain 0 . So by a finite irreducible root system we will mean a finite subset $\Delta$ of a finite dimensional vector space $X$ over $k$ such that $0 \in \Delta$ and $\Delta^{\times}:=\Delta \backslash\{0\}$ is a finite irreducible root system in $X$ in the usual sense (see [B2, Chap VI, $\S 1$, no. 1, Definition 1]). With this convention we will assume for the rest of this section that $\Delta$ is a finite irreducible root system in a finite dimensional vector space $X$ over $k$. Recall that $\Delta$ is said to be reduced if $2 \alpha \notin \Delta^{\times}$for $\alpha \in \Delta^{\times}$. If $\Delta$ is reduced then $\Delta$ has type $\mathrm{A}_{\ell}(\ell \geq 1), \mathrm{B}_{\ell}(\ell \geq 2), \mathrm{C}_{\ell}(\ell \geq 3), \mathrm{D}_{\ell}(\ell \geq 4), \mathrm{E}_{6}, \mathrm{E}_{7}, \mathrm{E}_{8}, \mathrm{~F}_{4}$, or $\mathrm{G}_{2}$, whereas if $\Delta$ is not reduced, $\Delta$ has type $\mathrm{BC}_{\ell}(\ell \geq 1)$ [B2, Chapter VI, $\S 4$ and 14]. We let

$$
\Delta_{\text {ind }}:=\{0\} \cup\left\{\alpha \in \Delta^{\times} \mid \frac{1}{2} \alpha \notin \Delta\right\},
$$

in which case $\Delta_{\text {ind }}$ is an irreducible reduced root system in $X$, and $\Delta_{\text {ind }}=\Delta$ if $\Delta$ is reduced. Also we let

$$
Q=Q(\Delta):=\operatorname{span}_{\mathbb{Z}}(\Delta)
$$

be the root lattice of $\Delta$.

If $L=\bigoplus_{(\alpha, \lambda) \in Q \times \Lambda} L_{\alpha}^{\lambda}$ is a $Q \times \Lambda$-graded algebra, then $L=\bigoplus_{\lambda \in \Lambda} L^{\lambda}$ is $\Lambda$-graded and $L=\bigoplus_{\alpha \in Q} L_{\alpha}$ is $Q$-graded, with

\footnotetext{
${ }^{4}$ We denote the centre of a Lie algebra $L$ by $Z(L)$. $L$ is called centreless if $Z(L)=0$.
} 


$$
L^{\lambda}=\bigoplus_{\alpha \in Q} L_{\alpha}^{\lambda} \text { for } \lambda \in \Lambda \quad \text { and } \quad L_{\alpha}=\bigoplus_{\lambda \in \Lambda} L_{\alpha}^{\lambda} \text { for } \alpha \in Q .
$$

Definition 3.1. $A$ Lie $\Lambda$-torus of type $\Delta$ is a $Q \times \Lambda$-graded Lie algebra $L=$ $\bigoplus_{(\alpha, \lambda) \in Q \times \Lambda} L_{\alpha}^{\lambda}$ over $k$ which satisfies:

(LT1) $L_{\alpha}=0$ for $\alpha \in Q \backslash \Delta$.

(LT2) (i) If $0 \neq \alpha \in \Delta$, then $L_{\alpha}^{0} \neq 0$.

(ii) If $0 \neq \alpha \in Q, \lambda \in \Lambda$ and $L_{\alpha}^{\lambda} \neq 0$, then there exist elements $e_{\alpha}^{\lambda} \in L_{\alpha}^{\lambda}$ and $f_{\alpha}^{\lambda} \in L_{-\alpha}^{-\lambda}$ such that

$$
L_{\alpha}^{\lambda}=\mathbb{F} e_{\alpha}^{\lambda}, \quad L_{-\alpha}^{-\lambda}=\mathbb{F} f_{\alpha}^{\lambda},
$$

and

$$
\left[\left[e_{\alpha}^{\lambda}, f_{\alpha}^{\lambda}\right], x_{\beta}\right]=\left\langle\beta, \alpha^{\vee}\right\rangle x_{\beta}
$$

for $x_{\beta} \in L_{\beta}, \beta \in Q$.

(LT3) $L$ is generated as an algebra by the spaces $L_{\alpha}, \alpha \in \Delta^{\times}$.

(LT4) $\Lambda$ is generated as a group by $\operatorname{supp}_{\Lambda}(L)$.

In that case the $Q$-grading of $L$ is called the root grading of $L$, and the $\Lambda$-grading of $L$ is called the external grading of $L$. If $\Delta$ has type $X_{\ell}$, we also say that $L$ has type $\mathrm{X}_{\ell}$.

Remark 3.2. Suppose $L$ is a Lie $\Lambda$-torus of type $\Delta$.

(i) It is shown in [ABFP2, Prop. 1.1.10] that $\operatorname{supp}_{Q}(L)$ equals either $\Delta$ or $\Delta_{\text {ind }}$.

(ii) It is sometimes convenient to assume the following additional axiom:

(LT5) $\operatorname{supp}_{Q}(L)=\Delta$.

Note that by (i), (LT5) holds automatically if $\Delta$ is reduced. Also, if (LT5) does not hold, then we can replace $\Delta$ by $\Delta_{\text {ind }}$, in which case (LT5) holds. Thus, when convenient there is no loss of generality in assuming (LT5).

In the study of Lie tori it is not convenient to fix a particular realization of the root system $\Delta$ or a particular identification of the group $\Lambda$ with $\mathbb{Z}^{n}$. For this reason, the following is a natural notion of isomorphism for Lie tori.

Definition 3.3. If $L$ is a Lie $\Lambda$-torus of type $\Delta$ with $Q=Q(\Delta)$ and $L^{\prime}$ is a Lie $\Lambda^{\prime}$ torus of type $\Delta^{\prime}$ with $Q^{\prime}=Q\left(\Delta^{\prime}\right), L$ and $L^{\prime}$ are said to be bi-isograded-isomorphic or bi-isomorphic for short, if there is an algebra isomorphism $\varphi: L \rightarrow L^{\prime}$, a group isomorphism $\varphi_{r}: Q \rightarrow Q$, and a group isomorphism $\varphi_{e}: \Lambda \rightarrow \Lambda^{\prime}$ such that

$$
\varphi\left(L_{\alpha}^{\lambda}\right)=L_{\varphi_{r}(\alpha)}^{\varphi_{e}(\lambda)}
$$

for $\alpha \in Q(\Delta)$ and $\lambda \in \Lambda$. In that case we write

$$
L \simeq_{b i} L^{\prime}
$$

and we call $\varphi$ a bi-isograded-isomorphism, or a bi-isomorphism for short. Since $\left\langle\operatorname{supp}_{Q}(L)\right\rangle=Q$ and $\left\langle\operatorname{supp}_{\Lambda}(L)\right\rangle=\Lambda$, the maps $\varphi_{r}$ and $\varphi_{e}$ are uniquely determined.

Remark 3.4. Suppose that $\varphi: L \rightarrow L^{\prime}$ is a bi-isomorphism as in Definition 3.3. We did not assume that $\varphi_{r}$ carries $\Delta$ onto $\Delta^{\prime}$. However, this holds automatically, if $L$ and $L^{\prime}$ satisfy (LT5). In particular, if $L$ and $L^{\prime}$ satisfy (LT5) and $\Delta^{\prime}=\Delta$, then $\varphi_{r} \in \operatorname{Aut}(\Delta)$. 
Definition 3.5. Suppose that $L$ is a centreless Lie $\Lambda$-torus of type $\Delta$. We set

$$
\mathfrak{g}=L^{0} \quad \text { and } \quad \mathfrak{h}=L_{0}^{0} .
$$

Then $\mathfrak{g}$ is a finite dimensional simple Lie algebra and $\mathfrak{h}$ is a splitting Cartan subalgebra of $\mathfrak{g}$ [N1, ABFP2]. Moreover, $\mathfrak{h}$ acts ad-diagonally on L. Furthermore, we can and do identify $\Delta$ with a root system in the dual space $\mathfrak{h}^{*}$ in such a way that $\Delta(\mathfrak{g}, \mathfrak{h})=\Delta_{\text {ind }}, \Delta(L, \mathfrak{h})=\Delta$ or $\Delta_{\text {ind }}$, and, for $\alpha \in Q$, the root space of $L$ relative to $\alpha$ is $L_{\alpha}$ [ABFP2, Prop. 1.2.2]. In particular, the type of the split simple Lie algebra $\mathfrak{g}$ is the type of the root system $\Delta_{\text {ind }}$. We call $(\mathfrak{g}, \mathfrak{h})$ the grading pair for $L .^{5}$

Remark 3.6 ([BN, Prop. 3.13]). Suppose that $L$ is a $\Lambda$-torus, and let $\operatorname{Cent}(L)$ be the centroid of $L .{ }^{6}$ Then, $\operatorname{Cent}(L)$ is $\Lambda$-graded, with $\operatorname{Cent}(L)^{\lambda}=\{c \in \operatorname{Cent}(L) \mid$ $c\left(L^{\mu}\right) \subseteq L^{\lambda+\gamma}$ for $\left.\mu \in \Lambda\right\}$. Let

$$
\Gamma(L):=\operatorname{supp}_{\Lambda}(\operatorname{Cent}(L))
$$

be the support of Cent $(L)$, in which case $\Gamma(L)$ is a subgroup of $\Lambda$ called the centroidal grading group of $L$. In that case, Cent $(L)$ is graded-isomorphic to the group algebra $\mathbb{F}[\Gamma]$ with its natural $\Gamma$-grading (and hence also with its $\Lambda$-grading).

Example 3.7 (The untwisted centreless Lie torus). Let $\mathfrak{g}$ be a finite dimensional split simple Lie algebra, let $\mathfrak{h}$ be a splitting Cartan subalgebra of $\mathfrak{g}$, let $\mathfrak{g}=\oplus_{\alpha \in \Delta} \mathfrak{g}_{\alpha}$ be the root space decomposition of $\mathfrak{g}$ relative to $\mathfrak{h}$, where $\Delta=\Delta(\mathfrak{g}, \mathfrak{h})$. Set $L=\mathfrak{g} \otimes \mathbb{F}[\Lambda]$ with $Q \times \Lambda$-grading given by $L_{\alpha}^{\lambda}=\mathfrak{g}_{\alpha} \otimes \mathbb{F}[\Lambda]^{\lambda}$ for $\alpha \in Q, \lambda \in \Lambda$. Then $L$ is a centreless Lie $\Lambda$-torus of type $\Delta$ which we call the untwisted centreless Lie $\Lambda$-torus of type $\Delta .^{7}$ The grading pair for $L$ is $(\mathfrak{g} \otimes 1, \mathfrak{h} \otimes 1)$. The centroid of $L$ consists of multiplications by elements of $\mathbb{F}[\Lambda]$, so $\Gamma(L)=\Lambda$.

In later sections, we will need the following lemma about bi-isomorphisms of centerless Lie tori of the same type.

Lemma 3.8. Let $L$ be a Lie $\Lambda$-torus of type $\Delta$ with grading pair $(\mathfrak{g}, \mathfrak{h})$, and let $L^{\prime}$ be a Lie $\Lambda^{\prime}$-torus of type $\Delta$ with grading pair $\left(\mathfrak{g}^{\prime}, \mathfrak{h}^{\prime}\right)$. Let $\Pi=\left\{\alpha_{1}, \ldots, \alpha_{\ell}\right\}$ be a base for the root system $\Delta$, choose $0 \neq e_{i} \in \mathfrak{g}_{\alpha_{i}}, 0 \neq f_{i} \in \mathfrak{g}_{-\alpha_{i}}$ with $\left[\left[e_{i}, f_{i}\right], e_{i}\right]=2 e_{i}$, and choose $0 \neq e_{i}^{\prime} \in \mathfrak{g}_{\alpha_{i}}^{\prime}, 0 \neq f_{i}^{\prime} \in \mathfrak{g}_{-\alpha_{i}}^{\prime}$ with $\left[\left[e_{i}^{\prime}, f_{i}^{\prime}\right], e_{i}^{\prime}\right]=2 e_{i}^{\prime}$. Suppose there is a bi-isomorphism $\varphi: L \rightarrow L^{\prime}$ with $\varphi_{r}$ in the Weyl group of $\Delta$. (Here we are using the notation $\varphi_{r}$ and $\varphi_{e}$ of Definition 3.3.) Then, there exists a bi-isomorphism $\tilde{\varphi}: L \rightarrow L^{\prime}$ such that $\tilde{\varphi}_{e}=\varphi_{e}, \tilde{\varphi}_{r}=1, \tilde{\varphi}\left(e_{i}\right)=e_{i}^{\prime}$ and $\tilde{\varphi}\left(f_{i}\right)=f_{i}^{\prime}$ for all $i$.

Proof. Since $\varphi_{r}$ is in the Weyl group of $\Delta$, there exists an automorphism $\eta$ of $\mathfrak{g}$ such that $\eta(\mathfrak{h})=\mathfrak{h}$ and $\eta\left(\mathfrak{g}_{\alpha}\right)=\mathfrak{g}_{\varphi_{r}(\alpha)}$ for all $\alpha \in \Delta$ [B3, Theorem 2(ii), Ch. VIII, $\S 2$, no. 2]. Moreover, $\eta$ can be chosen in the form $\eta=\prod_{i} \exp \left(\operatorname{ad}_{\mathfrak{g}}\left(x_{i}\right)\right)$, where each $x_{i}$ is in a root space of $\mathfrak{g}$ corresponding to a nonzero root. Thus each $\operatorname{ad}_{L}\left(x_{i}\right)$ is

\footnotetext{
${ }^{5}$ In [N1], the grading pair is defined for not necessarily centreless Lie tori . For centreless Lie tori the two definitions are equivalent [ABFP2, Prop. 1.2.1].

${ }^{6}$ Recall that the centroid of an algebra is the associative algebra of all endomorphisms of the algebra that commute with all left and right multiplications.

7 The universal central extension of $\mathfrak{g} \otimes \mathbb{F}[\Lambda]$ is called the toroidal Lie algebra [MRY], which is one of the origins of the term Lie torus.
} 
nilpotent and so we may extend $\eta$ to $\eta=\prod_{i} \exp \left(\operatorname{ad}_{L}\left(x_{i}\right)\right)$. Then $\eta: L \rightarrow L$ is a bi-isomorphism with $\eta_{e}=1$ and $\eta_{r}=\varphi_{r}$. So replacing $\varphi$ by $\varphi \eta^{-1}$, we can assume that $\varphi_{r}=1$. Thus, $\varphi\left(\mathfrak{g}_{\alpha_{i}}\right)=\varphi\left(\mathfrak{g} \cap L_{\alpha_{i}}\right)=\mathfrak{g}^{\prime} \cap L_{\alpha_{i}}^{\prime}=\mathfrak{g}_{\alpha_{i}}^{\prime}$ for all $i$. So $\varphi\left(e_{i}\right)=a_{i} e_{i}^{\prime}$ and $\varphi\left(f_{i}\right)=a_{i}^{-1} f_{i}^{\prime}$ for some $a_{i} \in \mathbb{F}^{\times}$. Choose a group homomorphism $\rho: Q \rightarrow \mathbb{F}^{\times}$ such that $\rho\left(\alpha_{i}\right)=a_{i}$ for all $i$, and define $\tau \in \operatorname{Aut}(L)$ by $\tau\left(x_{\alpha}\right)=\rho(\alpha) x_{\alpha}$ for $x_{\alpha} \in L_{\alpha}$, $\alpha \in Q$. Then $\tau: L \rightarrow L$ is a bi-isomorphism with $\tau_{e}=1$ and $\tau_{r}=1$. So $\varphi \tau^{-1}$ is the required $\tilde{\varphi}$.

\section{The construction of EALAs from Lie tori}

In [BGK], Berman, Gao and Krylyuk gave a construction of a family of EALAs starting from a centreless Lie tori of type $\mathrm{A}_{r}, r \geq 3$. In [N2], Neher simplified this construction and extended it to all types. Since we will be working with this construction in some detail, we give a careful description of it in this section. Facts that we note without reference are either straightforward or can be found in [N1, N2].

We first need to establish some notation and assumptions. Throughout the section, we assume that $L$ is a centreless Lie $\Lambda$-torus of type $\Delta$ and we let $Q=Q(\Delta)$.

Let

$$
\Gamma=\Gamma(L)
$$

be the centroidal grading group of $L$. We identify $\operatorname{Cent}(L)=\mathbb{F}[\Gamma]$ (see Remark 3.6) by fixing a basis $\left\{t^{\gamma}\right\}_{\gamma \in \Gamma}$ for $\operatorname{Cent}(L)$ satisfying $t^{\gamma} t^{\delta}=t^{\gamma+\delta}$.

Next let $\operatorname{Hom}(\Lambda, \mathbb{F})$ be the group of group homomorphisms from $\Lambda$ into $\mathbb{F}$. Then for $\theta \in \operatorname{Hom}(\Lambda, \mathbb{F})$, let $\partial_{\theta} \in \operatorname{Der}(L)$ be the degree derivation defined by

$$
\partial_{\theta}\left(x^{\lambda}\right)=\theta(\lambda) x^{\lambda}
$$

for $x^{\lambda} \in L^{\lambda}, \lambda \in \Lambda$. Put

$$
\mathcal{D}=\left\{\partial_{\theta} \mid \theta \in \operatorname{Hom}(\Lambda, \mathbb{F})\right\} \quad \text { and } \quad \operatorname{CDer}(L)=\operatorname{Cent}(L) \mathcal{D} .
$$

Then $\operatorname{CDer}(L)$ is a subalgebra of the Lie algebra $\operatorname{Der}(L)$ with product given by

$$
\left[t^{\gamma_{1}} \partial_{\theta_{1}}, t^{\gamma_{2}} \partial_{\theta_{2}}\right]=t^{\gamma_{1}+\gamma_{2}}\left(\theta_{1}\left(\gamma_{2}\right) \partial_{\theta_{2}}-\theta_{2}\left(\gamma_{1}\right) \partial_{\theta_{1}}\right)
$$

and $\operatorname{CDer}(L)$ is $\Gamma$-graded with $\operatorname{CDer}(L)^{\gamma}=\operatorname{Cent}(L)^{\gamma} \mathcal{D}$ for $\gamma \in \Gamma$.

Since $L$ is a centreless Lie torus, there is a nondegenerate invariant $\Lambda$-graded form $(\mid)$ on $L$, and that form is unique up to multiplication by a nonzero scalar [Y6, Thm. 2.2 and 7.1]. We fix a choice of $(\mid)$ on $L$. Then, since $L$ is perfect and $(\mid)$ is invariant we have

$$
(c(x), y)=(x, c(y))
$$

for $x, y \in L, c \in \operatorname{Cent}(L)$. Now let $\operatorname{SCDer}(L)$ be the subalgebra of $\operatorname{CDer}(L)$ consisting of the derivations in $\operatorname{CDer}(L)$ that are skew relative to the form $(\mid)$ ). (This subalgebra does not depend on the choice of the form $(\mid)$.) Then, $\operatorname{SCDer}(L)$ is a $\Gamma$-graded subalgebra of $\operatorname{CDer}(L)$ with

$$
\operatorname{SCDer}(L)^{\gamma}=t^{\gamma}\left\{\partial_{\gamma} \in \mathcal{D} \mid \theta(\gamma)=0\right\}
$$

for $\gamma \in \Gamma \cdot \operatorname{SCDer}(L)$ is called the algebra of skew-centroidal derivations of $L$. 
Now suppose that $D=\oplus_{\gamma \in \Gamma} D_{\gamma}$ is a $\Gamma$-graded subalgebra of $\operatorname{SCDer}(L)$. The graded-dual space of $D$ is the subspace $D^{\mathrm{gr} *}=\oplus_{\gamma \in \Gamma}\left(D_{\gamma}\right)^{*}$ of $D^{*}$, where $\left(D_{\gamma}\right)^{*}$ is embedded in $D^{*}$ by letting its elements act trivially on $D_{\delta}$ for $\delta \neq \gamma$ We let

$$
C=D^{\mathrm{gr} *}
$$

and we give the vector space $C$ a $\Gamma$-grading by setting

$$
C_{\gamma}=\left(D_{-\gamma}\right)^{*}
$$

With this grading, $C$ is a $\Gamma$-graded $D$-module by means of the contragradient action . given by

$$
(d \cdot f)(e)=-f([d, e]),
$$

for $d, e \in D, f \in C$. We also regard $C$ as a $L$-module with trivial action. Now define $\sigma_{D}: L \times L \rightarrow C$ by

$$
\sigma_{D}(x, y)(d)=(d x \mid y)
$$

Then $\sigma_{D}$ is a $\Gamma$-graded 2-cocycle for $L$ with values in the trivial $L$-module $C$. $^{8}$ Finally, let ev $: \Lambda \rightarrow C_{0}$ denote the evaluation map defined by

$$
(\operatorname{ev}(\lambda))\left(\partial_{\theta}\right)=\theta(\lambda)
$$

for $\lambda \in \Lambda$ and $\partial_{\theta} \in D_{0} \subseteq \operatorname{SCDer}(L)^{0}=\mathcal{D}$.

With this background we now present the construction.

Construction 4.1 ([N2]). Let $L$ be a centreless Lie $\Lambda$-torus of type $\Delta$. In order to construct an EALA from $L$ we need two additional ingredients. We assume that

(4.1a) $D$ is a $\Gamma$-graded subalgebra of $\operatorname{SCDer}(L)$ such that the map ev : $A \rightarrow C_{0}$ is injective, where $\Gamma=\Gamma(L)$ and $C=D^{\text {gr* }}$.

(4.1b) $\tau: D \times D \rightarrow C$ is a $\Gamma$-graded invariant 2-cocycle of $D$ with values in the $D$-module $C$ and $\tau\left(D_{0}, D\right)=0$.

Note that the assumption in (4.1b) that $\tau$ is invariant means that

$$
\tau\left(d_{1}, d_{2}\right)\left(d_{3}\right)=\tau\left(d_{2}, d_{3}\right)\left(d_{1}\right)
$$

for $d_{i} \in D$. Let

$$
E=E(L, D, \tau)=D \oplus L \oplus C,
$$

where $C=D^{\mathrm{gr} *} \cdot{ }^{9}$ We identify $D, L$ and $C$ naturally as subspaces of $E$. Define a product $[,]_{E}$ on $E$ by

$$
\begin{aligned}
{\left[d_{1}+l_{1}+f_{1}, d_{2}+l_{2}+f_{2}\right]_{E}=\left[d_{1}, d_{2}\right] } & +\left(\left[l_{1}, l_{2}\right]+d_{1}\left(l_{2}\right)-d_{2}\left(l_{1}\right)\right) \\
& +\left(d_{1} \cdot f_{2}-d_{2} \cdot f_{1}+\sigma_{D}\left(l_{1}, l_{2}\right)+\tau\left(d_{1}, d_{2}\right)\right)
\end{aligned}
$$

\footnotetext{
${ }^{8}$ In this section and in $\S 6$ we use the terminology (cochain, cocycle, coboundary and extension) from the cohomology of Lie algebras. See for example [B1, Chapter I, Exercise 12 for $\S 3]$.

${ }^{9}$ We have changed the order used in [N2] of the components of $E$. This is convenient in the proof of Corollary 6.3 below.
} 
for $d_{i} \in D, l_{i} \in L, f_{i} \in C$. Then, $E$ is a $\Lambda$-graded algebra with the direct sum grading. Next we extend the bilinear form $(\mid)$ on $L$ to a graded bilinear form $(\mid)$ on $E$ by defining:

$$
\left(d_{1}+l_{1}+f_{1} \mid d_{2}+l_{2}+f_{2}\right)=\left(l_{1} \mid l_{2}\right)+f_{1}\left(d_{2}\right)+f_{2}\left(d_{1}\right) .
$$

Finally let

$$
H=D_{0} \oplus \mathfrak{h} \oplus C_{0} .
$$

in $E$, where $(\mathfrak{g}, \mathfrak{h})=\left(L^{0}, L_{0}^{0}\right)$ is the grading pair for $L$. Thus we have constructed a triple $((E(L, D, \tau),(\mid), H)$, which we often denote simply by $E(L, D, \tau)$.

Neher has announced the following fundamental result on this construction:

Theorem 4.2 ([N2, Thm. 6]). (i) If $L$ is a centreless Lie $\Lambda$-torus, D satisfies (4.1a) and $\tau$ satisfies (4.1b), then $E(L, D, \tau)$ is an extended affine Lie algebra of nullity $\operatorname{rank}(\Lambda)$.

(ii) If $E$ is an $E A L A$, then $E$ is isomorphic as an $E A L A$ to $E(L, D, \tau)$ for some $L, D$ and $\tau$ as in (i).

Remark 4.3. (i) It is easy to check that the EALA $E(L, D, \tau)$ in part (i) of the theorem does not depend, up to EALA isomorphism, on the choice of the form $(\mid)$ on $L$.

(ii) Neher actually states more than we've stated in part (ii) of the theorem. Indeed, given an EALA $E$, then $E_{\mathrm{cc}}$, with a suitable grading, is a Lie torus satisfying (LT5) and $E$ is isomorphic as an EALA to $E\left(E_{\mathrm{cc}}, D, \tau\right)$ for some $D$ and $\tau$ as in (i) [N2, Thm. 6(ii)].

(iii) In particular, we can always choose $L$ in part (ii) of the theorem satisfying (LT5). In that case, the type of $E$ and $L$ are the same.

(iv) The Lie torus $L$ in part (ii) of the theorem is not uniquely determined up to bi-isomorphism. However, it is uniquely determined up to isotopy, a fact that has motivated our work on this topic. We discuss this in detail in $\S 6$ below.

Remark 4.4. Suppose $L$ is a centreless Lie torus, $\Gamma=\Gamma(L)$, and $D$ is a $\Gamma$-graded subalgebra of $\operatorname{SCDer}(L)$. Let $M:=D \oplus L$ and identify $D$ and $L$ naturally as subspaces of $M$. Then, $M$ is a $\Lambda$-graded Lie algebra with product [, ] given by

$$
\left[d_{1}+l_{1}, d+l_{2}\right]=\left[d_{1}, d_{2}\right]+\left(\left[l_{1}, l_{2}\right]+d_{1}\left(l_{2}\right)-d_{2}\left(l_{1}\right)\right),
$$

and $C$ is an $M$-module via $(d+l) \cdot f=d \cdot f$. The cocycles $\sigma_{D}$ and $\tau$ can be extended to cocycles for the Lie algebra $M$ with values in the $M$-module $C$ by letting $\sigma_{D}(D, M)=\sigma_{D}(M, D)=0$ and $\tau(L, M)=\tau(M, L)=0$. If $D$ and $\tau$ satisfy (4.1a) and $(4.1 \mathrm{~b})$, then as a Lie algebra $E(L, D, \tau)$ is equal to the extension $\operatorname{Ext}\left(M, C, \sigma_{D}+\right.$ $\tau)$ of $M$ by $C$ using the cocycle $\sigma_{D}+\tau$.

If $L$ is a centreless Lie torus, we let

$$
\mathcal{P}(L)=\{(D, \tau) \mid D \text { satisfies (4.1a) and } \tau \text { satisfies (4.1b) }\} .
$$

We note $\mathcal{P}(L)$ is nonempty since in particular $(\operatorname{SCDer}(L), 0) \in \mathcal{P}(L)$. With this notation Theorem 4.2(i) tells us that Construction 4.1 builds a family

$$
\{E(L, D, \tau)\}_{(D, \tau) \in \mathcal{P}(L)}
$$

of EALAs from L. Also, Theorem 4.2(ii) tells us that any EALA $E$ occurs (up to isomorphism of EALAs) in the family $\{E(L, D, \tau)\}_{(D, \tau) \in \mathcal{P}(L)}$ constructed from some centreless Lie torus $L$. 


\section{Isotopy for Lie tori}

In this section, we recall the definition of isotopy from [ABFP2]. Throughout the section, we assume that $L$ is a Lie $\Lambda$-torus of type $\Delta$ and we let $Q=Q(\Delta)$.

If $\alpha \in Q$, we let

$$
\Lambda_{\alpha}=\Lambda_{\alpha}(L):=\left\{\lambda \in \Lambda \mid L_{\alpha}^{\lambda} \neq 0\right\} .
$$

We call $\Lambda_{\alpha}$ the $\Lambda$-support of $L$ at $\alpha$. Note that $0 \in \Lambda_{\alpha}$ if $0 \neq \alpha \in \Delta_{\text {ind }}$, by (LT2)(ii).

Definition 5.1. Suppose that $s \in \operatorname{Hom}(Q, \Lambda)$, where $\operatorname{Hom}(Q, \Lambda)$ is the group of group homomorphisms from $Q$ into $\Lambda$. We define a new $Q \times \Lambda$-graded Lie algebra $L^{(s)}$ as follows. As a Lie algebra, $L^{(s)}=L$. The grading on $L^{(s)}$ is given by

$$
\left(L^{(s)}\right)_{\alpha}^{\lambda}=L_{\alpha}^{\lambda+s(\alpha)}
$$

for $\alpha \in Q, \lambda \in \Lambda$.

The following necessary and sufficient conditions for $L^{(s)}$ to be a Lie torus are obtained in [ABFP2, Prop. 2.2.3].

Proposition 5.2. Let $s \in \operatorname{Hom}(Q, \Lambda)$, and let $\Pi$ be a base for the root system $\Delta$. The following statements are equivalent:

(a) $L^{(s)}$ is a Lie torus.

(b) $s(\alpha) \in \Lambda_{\alpha}$ for all $0 \neq \alpha \in \Delta_{\text {ind }}$.

(c) $s(\alpha) \in \Lambda_{\alpha}$ for all $\alpha \in \Pi$.

Definition 5.3 (Isotopes of Lie tori). Suppose that $s \in \operatorname{Hom}(Q, \Lambda)$. If s satisfies the equivalent conditions in Proposition 5.2, we say that $s$ is admissible for $L$. In that case, we call the Lie torus $L^{(s)}$ the $s$-isotope of $L$.

Remark 5.4. Suppose that $\Pi=\left\{\alpha_{1}, \ldots, \alpha_{r}\right\}$ is a base for $\Delta$. By Proposition 5.2, to specify an admissible $s \in \operatorname{Hom}(Q, \Lambda)$ for $L$, and hence an isotope $L^{(s)}$ of $L$, one can arbitrarily choose $\lambda_{i} \in \Lambda_{\alpha_{i}}$ for $1 \leq i \leq r$, and then define $s \in \operatorname{Hom}(Q, \Lambda)$ with $s\left(\alpha_{i}\right)=\lambda_{i}$ for $1 \leq i \leq r$.

Definition 5.5. Suppose that $L$ is a Lie $\Lambda$-torus of type $\Delta$ and $L^{\prime}$ is a Lie $\Lambda^{\prime}$-torus of type $\Delta^{\prime}$. We say that $L$ is isotopic to $L^{\prime}$, written $L \sim L^{\prime}$, if some isotope $L^{(s)}$ of $L$ is bi-isomorphic to $L^{\prime}$.

Using the facts that $L^{(0)}=L,\left(L^{(s)}\right)^{(t)}=L^{(s+t)}$ and $\left(L^{(s)}\right)^{(-s)}=L$ as $Q \times \Lambda$ graded algebras for $s, t \in \operatorname{Hom}(Q, \Lambda)$, it is shown in [ABFP2, §2] that isotopy is an equivalence relation on the class of Lie tori.

\section{Isotopy in the theory of EALAs}

As promised at the end of $\S 4$, we now describe the role that isotopy plays in the structure theory of EALAs. Our first theorem on this topic was actually the starting point of our investigation of isotopy. 
Theorem 6.1. Suppose $L$ is a centreless Lie $\Lambda$-torus of type $\Delta$ and $L^{\prime}$ is a centreless Lie $\Lambda^{\prime}$-torus of type $\Delta^{\prime}$. If some member of the family $\{E(L, D, \tau)\}_{(D, \tau) \in \mathcal{P}(L)}$ is isomorphic as an EALA to some member of the family $\left\{E\left(L^{\prime}, D^{\prime}, \tau^{\prime}\right)\right\}_{\left(D^{\prime}, \tau^{\prime}\right) \in \mathcal{P}\left(L^{\prime}\right)}$, then $L$ is isotopic to $L^{\prime}$.

Proof. Let $(\mathfrak{g}, \mathfrak{h})$ be the grading pair of $L$. Since $\Delta(L, \mathfrak{h})=\Delta$ or $\Delta_{\text {ind }}$ (see Definition $3.5)$, we can if necessary replace $\Delta$ by $\Delta_{\text {ind }}$ so that

$$
\Delta(L, \mathfrak{h})=\Delta .
$$

Suppose now that $E=E(L, D, \tau)$, where $(D, \tau) \in \mathcal{P}(L)$. We use the notation $Q, C$, ev, $H$ from Construction 4.1. Recall that $H=D_{0} \oplus \mathfrak{h} \oplus C$, so we can identify

$$
H^{*}=\left(D_{0}\right)^{*} \oplus \mathfrak{h}^{*} \oplus\left(C_{0}\right)^{*}=C_{0} \oplus \mathfrak{h}^{*} \oplus D_{0} .
$$

We use ev to identify $\Lambda$ with a subgroup of $C_{0} \subseteq H^{*}$.

Let $R$ be the set of roots of $E$ and let $R^{\times}$be the set of nonisotropic roots in $R$. Then by $[\mathrm{N} 2, \S 5]$, we have

$$
R^{\times}=\cup_{\alpha \in \Delta \times}\left(\Lambda_{\alpha}+\alpha\right) \subseteq R \subseteq \Lambda \oplus Q,
$$

where $\Lambda_{\alpha}=\Lambda_{\alpha}(L)$. It follows from this fact and (LT2)(i) that

$$
\langle R\rangle=\Lambda \oplus Q .
$$

Now by $[\mathrm{N} 2, \S 5]$, we have

$$
E_{\lambda+\alpha}=L_{\alpha}^{\lambda}
$$

for $\lambda \in \Lambda, 0 \neq \alpha \in Q$. It is easy to show using this equation, (7) and (LT3) that the core of $E$ (see Definition 2.1(ii)) is given by

$$
E_{\mathrm{c}}=L \oplus C .
$$

(In fact this is implicit in [N2].) Hence, since $L$ is centreless, we have

$$
Z\left(E_{\mathrm{c}}\right)=C
$$

Next, let $E^{\prime}=E\left(L^{\prime}, D^{\prime}, \tau^{\prime}\right)$, where $\left(D^{\prime}, \tau^{\prime}\right) \in \mathcal{P}\left(L^{\prime}\right)$, and we use all of the above notation (with primes added) for $L^{\prime}$ and $E^{\prime}$.

Suppose that $\chi: E \rightarrow E^{\prime}$ is an EALA isomorphism. So

$$
\chi(H)=H^{\prime},
$$

and $\chi$ preserves the forms up multiplication by a nonzero scalar. It follows that $\chi\left(E_{\mathrm{c}}\right)=E_{\mathrm{c}}^{\prime}$. So, by (10) and (11) (and the corresponding primed equations), we have

$$
\chi(L \oplus C)=L^{\prime} \oplus C^{\prime} \quad \text { and } \quad \chi(C)=C^{\prime} .
$$

Thus, there exists a unique Lie algebra isomorphism $\varphi: L \rightarrow L^{\prime}$ with

$$
\chi(l) \equiv \varphi(l) \quad\left(\bmod C^{\prime}\right)
$$

for $l \in L$. It then follows that

$$
\chi^{-1}\left(l^{\prime}\right) \equiv \varphi^{-1}\left(l^{\prime}\right) \quad(\bmod C)
$$


for $l^{\prime} \in L^{\prime}$ So

Now, by (12), we have $\varphi(\mathfrak{h}) \subseteq\left(H^{\prime} \oplus C^{\prime}\right) \cap L^{\prime}=\mathfrak{h}^{\prime}$, and similarly $\varphi^{-1}\left(\mathfrak{h}^{\prime}\right) \subseteq \mathfrak{h}$.

$$
\varphi(\mathfrak{h})=\mathfrak{h}^{\prime} .
$$

Hence, if $h \in \mathfrak{h}, \chi(h)-\varphi(h) \in H^{\prime} \cap C^{\prime}=C_{0}^{\prime}$, so

$$
\chi(h) \equiv \varphi(h) \quad\left(\bmod C_{0}^{\prime}\right) .
$$

Also, $\chi\left(C_{0}\right) \subseteq H^{\prime} \cap C^{\prime}=C_{0}^{\prime}$ and similarly $\chi^{-1}\left(C_{0}^{\prime}\right) \subseteq C_{0}$. So

$$
\chi\left(C_{0}\right)=C_{0}^{\prime} .
$$

But then, by (13), (14) and (15), $\chi\left(\mathfrak{h} \oplus C_{0}\right) \subseteq \mathfrak{h}^{\prime} \oplus C_{0}^{\prime}$; so using the corresponding property for $\chi^{-1}$, we have

$$
\chi\left(\mathfrak{h} \oplus C_{0}\right)=\mathfrak{h}^{\prime} \oplus C_{0}^{\prime} .
$$

Next define $\hat{\chi}: H^{*} \rightarrow H^{\prime *}$ by

$$
(\hat{\chi}(\rho))(\chi(t))=\rho(t)
$$

for $\rho \in H^{*}, t \in H$. ( $\hat{\chi}$ is the inverse dual of $\left.\chi\right|_{H}: H \rightarrow H^{\prime}$.) Similarly, define $\hat{\varphi}: \mathfrak{h}^{*} \rightarrow \mathfrak{h}^{\prime *}$ by

$$
(\hat{\varphi}(\alpha))(\varphi(h))=\alpha(h)
$$

for $\alpha \in \mathfrak{h}^{*}, h \in \mathfrak{h}$. Then, using $R=\Delta(E, H)$ and $\Delta=\Delta(L, \mathfrak{h})$ (and the corresponding primed equations), we see that

$$
\hat{\chi}(R)=R^{\prime} \quad \text { and } \quad \hat{\varphi}(\Delta)=\Delta^{\prime} .
$$

Thus, since $\langle R\rangle=\Lambda \oplus Q$ and $\langle\Delta\rangle=Q$, we have

$$
\begin{gathered}
\hat{\chi}(\Lambda \oplus Q)=\Lambda^{\prime} \oplus Q^{\prime}, \\
\hat{\varphi}(Q)=Q^{\prime} .
\end{gathered}
$$

Let $\alpha \in Q$, and write, using (17), $\hat{\chi}(\alpha)=\lambda^{\prime}+\alpha^{\prime}$, where $\lambda^{\prime} \in \Lambda^{\prime}, \alpha^{\prime} \in Q^{\prime}$. Then, $\left(\lambda^{\prime}+\alpha^{\prime}\right)(\chi(h))=\alpha(h)$ for $h \in \mathfrak{h}$ (in fact for $\left.h \in H\right)$. Thus, by $(14),\left(\lambda^{\prime}+\alpha^{\prime}\right)(\varphi(h))=$ $\alpha(h)$, so $\alpha^{\prime}(\varphi(h))=\alpha(h)$ for $h \in \mathfrak{h}$. Hence, $\alpha^{\prime}=\hat{\varphi}(\alpha)$. Thus, since $\hat{\varphi}$ is invertible,

$$
\hat{\chi}(\alpha)=s^{\prime}(\hat{\varphi}(\alpha))+\hat{\varphi}(\alpha)
$$

for $\alpha \in Q$ and some $s^{\prime} \in \operatorname{Hom}\left(Q^{\prime}, \Lambda^{\prime}\right)$.

Next let $\lambda \in \Lambda$, and as above write $\hat{\chi}(\lambda)=\lambda^{\prime}+\alpha^{\prime}$, where $\lambda^{\prime} \in \Lambda^{\prime}, \alpha^{\prime} \in Q^{\prime}$. Then, $\left(\lambda^{\prime}+\alpha^{\prime}\right)\left(\chi\left(\mathfrak{h}+C_{0}\right)\right)=\lambda\left(\mathfrak{h}+C_{0}\right)=0$. Hence, by $(16),\left(\lambda^{\prime}+\alpha^{\prime}\right)\left(\mathfrak{h}^{\prime}+C_{0}^{\prime}\right)=0$, so $\alpha^{\prime}=0$. Thus, $\hat{\chi}(\Lambda) \subseteq \Lambda^{\prime}$ and similarly $\hat{\chi}^{-1}\left(\Lambda^{\prime}\right)=\widehat{\chi^{-1}}\left(\Lambda^{\prime}\right) \subseteq \Lambda$. So

$$
\hat{\chi}(\Lambda)=\Lambda^{\prime} .
$$

Using (18) and (20), we let $\varphi_{r}=\left.\hat{\varphi}\right|_{Q}: Q \rightarrow Q^{\prime}$ and $\varphi_{e}=\left.\hat{\chi}\right|_{\Lambda}: \Lambda \rightarrow \Lambda^{\prime}$. Then, using (9) and (19), we have, for $0 \neq \alpha \in Q, \lambda \in \Lambda$,

$$
\begin{aligned}
\chi\left(L_{\alpha}^{\lambda}\right) & =\chi\left(E_{\lambda+\alpha}\right)=E_{\hat{\chi}(\lambda+\alpha)}^{\prime}=E_{\hat{\chi}(\lambda)+s^{\prime}(\hat{\varphi}(\alpha))+\hat{\varphi}(\alpha)}^{\prime} \\
& =\left(L^{\prime}\right)_{\hat{\varphi}(\alpha)}^{\hat{\chi}(\lambda)+s^{\prime}(\hat{\varphi}(\alpha))}=\left(\left(L^{\prime}\right)^{\left(s^{\prime}\right)}\right)_{\varphi_{r}(\alpha)}^{\varphi_{e}(\lambda)} .
\end{aligned}
$$


So by (12), we have

$$
\varphi\left(L_{\alpha}^{\lambda}\right)=\left(\left(L^{\prime}\right)^{\left(s^{\prime}\right)}\right)_{\varphi_{r}(\alpha)}^{\varphi_{e}(\lambda)}
$$

for $0 \neq \alpha \in Q, \lambda \in \Lambda$. But then, by (LT3), this equation holds for all $\alpha \in Q, \lambda \in \Lambda$. Therefore, since $L$ is a Lie $\Lambda$-torus of type $\Delta$, so is $\left(L^{\prime}\right)^{\left(s^{\prime}\right)}$. Hence $s^{\prime}$ is admissible for $L^{\prime}$, and $\varphi$ is a bi-isomorphism of $L$ onto $\left(L^{\prime}\right)^{\left(s^{\prime}\right)}$.

We next consider the relationship between the family of EALAs constructed from a Lie torus $L$ and the family of EALAs constructed from an isotope of $L$. For this purpose, we assume now that $L$ is a centreless Lie $\Lambda$-torus of type $\Delta, Q=Q(\Delta)$, $s \in \operatorname{Hom}(Q, \Lambda)$ is admissible for $L$, and $L^{(s)}$ is the $s$-isotope of $L$.

We fix a nondegenerate $\Lambda$-graded invariant symmetric bilinear form ( | ) on $L$ and we use that same form on $L^{(s)}$.

Let $\partial_{\theta}^{(s)}$ be the degree derivation of $L^{(s)}$ determined by $\theta \in \operatorname{Hom}(\Lambda, \mathbb{F})$. Define the $\Lambda$-graded Cent $(L)$-module isomorphism

$$
\Psi: \operatorname{CDer}(L) \rightarrow \operatorname{CDer}\left(L^{(s)}\right) \text { by } \Psi\left(c \partial_{\theta}\right)=c \partial_{\theta}^{(s)} .
$$

Using (2) we see that $\Psi$ is also a Lie algebra isomorphism. Let $h_{\theta} \in \mathfrak{h}$ be given by

$$
\alpha\left(h_{\theta}\right)=\theta(s(\alpha))
$$

for $\alpha \in Q$, and define the $\Lambda$-graded Cent $(L)$-module monomorphism

$$
\Omega: \operatorname{CDer}(L) \rightarrow L \quad \text { by } \quad \Omega\left(c \partial_{\theta}\right)=c h_{\theta}
$$

for $c \in \operatorname{Cent}(L)$. For $l \in\left(L^{(s)}\right)_{\alpha}^{\lambda}=L_{\alpha}^{\lambda+s(\alpha)}$, we have $\partial_{\theta}(l)=\theta(\lambda+s(\alpha)) l=\partial_{\theta}^{(s)}(l)+$ $\left[h_{\theta}, l\right]$. Thus,

$$
\partial_{\theta}^{(s)}=\partial_{\theta}-\operatorname{ad}_{L}\left(h_{\theta}\right) \quad \text { and } \quad \Psi(d)=d-\operatorname{ad}_{L}(\Omega(d))
$$

for $d \in \operatorname{CDer}(L)$. Since the derivations in $\operatorname{ad}_{L}(L)$ are skew, we see that $\Psi$ maps $\operatorname{SCDer}(L)$ into $\operatorname{SCDer}\left(L^{(s)}\right)$. We also note using $(2)$ that

$$
\begin{aligned}
\Omega\left(\left[t^{\gamma_{1}} \partial_{\theta_{1}}, t^{\gamma_{2}} \partial_{\theta_{2}}\right]\right) & =t^{\gamma_{1}} t^{\gamma_{2}}\left(\theta_{1}\left(\gamma_{2}\right) h_{\theta_{2}}-\theta_{2}\left(\gamma_{1}\right) h_{\theta_{1}}\right) \\
& =t^{\gamma_{1}} \partial_{\theta_{1}}\left(t^{\gamma_{2}} h_{\theta_{2}}\right)-t^{\gamma_{2}} \partial_{\theta_{2}}\left(t^{\gamma_{1}} h_{\theta_{1}}\right) \\
& =t^{\gamma_{1}} \partial_{\theta_{1}}\left(\Omega\left(t^{\gamma_{2}} \partial_{\theta_{2}}\right)\right)-t^{\gamma_{2}} \partial_{\theta_{2}}\left(\Omega\left(t^{\gamma_{1}} \partial_{\theta_{1}}\right)\right),
\end{aligned}
$$

for $\gamma_{i} \in \Gamma$ and $\theta_{i} \in \operatorname{Hom}(\Lambda, \mathbb{F})$, so $\Omega$ is a derivation of $\operatorname{CDer}(L)$ into $L$.

If $(D, \tau) \in \mathcal{P}(L)$, let

$$
D^{(s)}=\Psi(D),
$$

in which case $D^{(s)}$ is a $\Gamma$-graded subalgebra of $\operatorname{SCDer}(L)$. Also let

$$
\psi: D \rightarrow D^{(s)} \quad \text { and } \quad \omega: D \rightarrow L
$$

be the restrictions of $\Psi$ and $\Omega$ to $D$. Then $\psi$ is a $\Lambda$-graded Lie algebra isomorphism, $\omega$ is a $\Lambda$-graded vector space monomorphism, and

$$
\psi(d)=d-\operatorname{ad}_{L}(\omega(d))
$$

for $d \in D$. Finally let

$$
C^{(s)}=\left(D^{(s)}\right)^{\mathrm{gr} *},
$$


which is $\Gamma$-graded as in (3), and define a $\Lambda$-graded cochain

$$
\tau^{(s)}: D^{(s)} \times D^{(s)} \rightarrow C^{(s)} \quad \text { by } \quad \tau^{(s)}\left(\psi d_{1}, \psi d_{2}\right)\left(\psi d_{3}\right)=\tau\left(d_{1}, d_{2}\right)\left(d_{3}\right)
$$

for $d_{i} \in D$.

Theorem 6.2. Suppose that $L$ is a centreless Lie $\Lambda$-torus, $s \in \operatorname{Hom}(Q, \Lambda)$ is admissible for $L,(D, \tau) \in \mathcal{P}(L)$, and $\left(D^{(s)}, \tau^{(s)}\right)$ is defined by (21) and (23). Then $\left(D^{(s)}, \tau^{(s)}\right) \in \mathcal{P}\left(L^{(s)}\right)$. Moreover, $E=E(L, D, \tau)$ and $E^{(s)}=E\left(L^{(s)}, D^{(s)}, \tau^{(s)}\right)$ are isomorphic as EALAs. To be more precise, define $\chi: E \rightarrow E^{(s)}$ by

$$
\chi(x)=\psi(d)+(\omega(d)+l)+\eta(x)
$$

for $x=d+l+c \in E, d \in D, l \in L, f \in C$, where $\eta: E \rightarrow C^{(s)}$ is given by

$$
\eta(x)\left(\psi\left(d^{\prime}\right)\right)=f\left(d^{\prime}\right)-\left(l+\frac{1}{2} \omega(d) \mid \omega\left(d^{\prime}\right)\right)
$$

for $d^{\prime} \in D$. Then $\chi$ is an isometry and a A-graded Lie algebra isomorphism that maps $H$ to $H^{(s)}=D_{0}^{(s)} \oplus \mathfrak{h} \oplus C_{0}^{(s)}$.

Proof. Define $\hat{\psi}: C \rightarrow C^{(s)}$ by $\hat{\psi}(f)(\psi(d))=f(d)$ for $d \in D, f \in C$. Clearly, $\hat{\psi}$ is a $\Gamma$-graded vector space isomorphism. Let $\mathrm{ev}^{(s)}$ be the evaluation map for $L^{(s)}$. Then, for $\lambda \in \Lambda$ and $\partial_{\theta}^{(s)} \in D_{0}^{(s)}$, we have

$$
(\hat{\psi}(\operatorname{ev}(\lambda)))\left(\partial_{\theta}^{(s)}\right)=(\hat{\psi}(\operatorname{ev}(\lambda)))\left(\psi\left(\partial_{\theta}\right)\right)=(\operatorname{ev}(\lambda))\left(\partial_{\theta}\right)=\theta(\lambda)
$$

so $\left.\hat{\psi}\right|_{C_{0}} \circ \mathrm{ev}=\mathrm{ev}^{(s)}$. Hence, ev ${ }^{(s)}$ is injective; i.e., $D^{(s)}$ satisfies (4.1a).

It will be convenient to view $E$ as $\operatorname{Ext}\left(M, C, \sigma_{D}+\tau\right)$, the extension of $M=D \oplus L$ by $C$ using the cocycle $\sigma_{D}+\tau$ as in Remark 4.4. Let $M^{(s)}=D^{(s)} \oplus L$ and set

$$
\xi(d+l)=\psi(d)+\omega(d)+l
$$

for $d \in D, l \in L$. We claim

$$
\xi: M \rightarrow M^{(s)} \text { is a Lie algebra isomorphism. }
$$

Clearly, $\xi$ is bijective since $\psi$ is bijective. Since $\psi: D \rightarrow D^{(s)}$ is an isomorphism and $L$ is an ideal in $M^{(s)}$, the map $d+l \rightarrow \psi(d)+L=\xi(d+l)+L$ is a homomorphism $M \rightarrow M^{(s)} / L$. Thus, for $m_{1}, m_{2} \in M$,

$$
z:=\xi\left(\left[m_{1}, m_{2}\right]\right)-\left[\xi\left(m_{1}\right), \xi\left(m_{2}\right)\right] \in L .
$$

On the other hand, $\zeta: M \rightarrow \operatorname{Der}(L)$ given by $\zeta(m)=\left.\operatorname{ad}_{M}(m)\right|_{L}$ and similarly $\zeta^{(s)}: M^{(s)} \rightarrow \operatorname{Der}(L)$ are homomorphisms. Moreover,

$$
\zeta^{(s)}(\xi(d+l))=\psi(d)+\operatorname{ad}_{L}(\omega(d))+\operatorname{ad}_{L}(l)=d+\operatorname{ad}_{L}(l)=\zeta(d+l),
$$

so $\zeta^{(s)} \circ \xi=\zeta$. Thus,

$$
\operatorname{ad}_{L}(z)=\zeta^{(s)}(z)=\zeta\left(\left[m_{1}, m_{2}\right]\right)-\left[\zeta\left(m_{1}\right), \zeta\left(m_{2}\right)\right]=0 .
$$

Since $L$ is centreless, $z=0$ and $\xi$ is an isomorphism.

We also note that for $d, d^{\prime} \in D, l \in L, f \in C$, 


$$
\begin{aligned}
(\xi(d+l) \cdot \hat{\psi}(f))\left(\psi\left(d^{\prime}\right)\right) & =(\psi(d) \cdot \hat{\psi}(f))\left(\psi\left(d^{\prime}\right)\right)=-\hat{\psi}(f)\left(\left[\psi(d), \psi\left(d^{\prime}\right)\right]\right) \\
& =-f\left(\left[d, d^{\prime}\right]\right)=(d \cdot f)\left(d^{\prime}\right)=\hat{\psi}((d+l) \cdot f)\left(\psi\left(d^{\prime}\right),\right.
\end{aligned}
$$

SO

$$
\xi(m) \cdot \hat{\psi}(f)=\hat{\psi}(m \cdot f)
$$

for $m \in M, f \in C$.

Recall that the group $Z^{2}(M, C)$ of 2 -cocycles on $M$ with values in $C$ consists of all alternating bilinear maps $\mu: M \times M \rightarrow C$ such that

$$
\sum_{(i, j, k) \circlearrowleft}\left(\mu\left(\left[m_{i}, m_{j}\right], m_{k}\right)-m_{i} \cdot \mu\left(m_{j}, m_{k}\right)\right)=0
$$

for $m_{1}, m_{2}, m_{3} \in M$ where $(i, j, k) \circlearrowleft$ means $(i, j, k)$ is a cyclic permutation of $(1,2,3)$. We can use $\xi$ and $\hat{\psi}$ to transfer $\mu$ to $\tilde{\mu}: M^{(s)} \times M^{(s)} \rightarrow C^{(s)}$ with

$$
\tilde{\mu}\left(\xi\left(m_{1}\right), \xi\left(m_{2}\right)\right)=\hat{\psi}\left(\mu\left(m_{1}, m_{2}\right)\right) .
$$

Using (24) and (25), we see that $\rho(\mu)=\tilde{\mu}$ defines a group isomorphism $\rho$ : $Z^{2}(M, C) \rightarrow Z^{2}\left(M^{(s)}, C^{(s)}\right)$. Furthermore, the map $\xi \oplus \hat{\psi}: \operatorname{Ext}(M, C, \mu) \rightarrow$ $\operatorname{Ext}\left(M^{(s)}, C^{(s)}, \rho(\mu)\right)$ is a Lie algebra isomorphism.

Let $\tilde{\tau}=\rho(\tau)$. Then $\tilde{\tau}\left(M^{(s)}, L\right)=\tilde{\tau}(\xi(M), \xi(L))=\hat{\psi}(\tau(M, L))=0$ and thus also $\tilde{\tau}\left(L, M^{(s)}\right)=0$. Moreover, for $d_{1}, d_{2}, d_{3} \in D$, we have

$$
\tilde{\tau}\left(\psi\left(d_{1}\right), \psi\left(d_{2}\right)\right)\left(\psi\left(d_{3}\right)\right)=\hat{\psi}\left(\tau\left(d_{1}, d_{2}\right)\right)\left(\psi\left(d_{3}\right)\right)=\tau\left(d_{1}, d_{2}\right)\left(d_{3}\right),
$$

so $\tilde{\tau}$ is the extension of $\tau^{(s)}$ to $M^{(s)}$ and $\tau^{(s)}$ is a cocycle. The invariance of $\tau^{(s)}$ is immediate from the invariance of $\tau$, while the equation $\tau^{(s)}\left(D_{0}^{(s)}, D^{(s)}\right)=0$ is immediate from the definition of $\tau^{(s)}$ and $\psi\left(D_{0}\right)=D_{0}^{(s)}$. This shows $\left(D^{(s)}, \tau^{(s)}\right) \in$ $\mathcal{P}\left(L^{(s)}\right)$.

It remains to prove the statement about $\chi$. To do this it will be helpful for us to write an element $x=d+l+f \in E$, where $d \in D, l \in L, f \in C$, as a column vector

$$
x=\left[\begin{array}{l}
d \\
l \\
f
\end{array}\right] .
$$

Similarly we express elements of $E^{(s)}$ as column vectors. This notation allows us to write linear maps (for example from $E$ to $E^{(s)}$ ) as matrices.

Define

$$
\omega^{\#}: L \rightarrow C \text { by } \omega^{\#}(l)(d)=(l \mid \omega(d)) .
$$

We see that for $x=d+l+c \in E, d \in D, l \in L, f \in C$ and for $d^{\prime} \in D$, we have

$$
\eta(x)\left(\psi\left(d^{\prime}\right)\right)=\left(f-\omega^{\#}\left(l+\frac{1}{2} \omega(d)\right)\right)\left(d^{\prime}\right)=\hat{\psi}\left(f-\omega^{\#}\left(l+\frac{1}{2} \omega(d)\right)\right)\left(\psi\left(d^{\prime}\right)\right),
$$

so $\eta(x)=\hat{\psi}(f)-\left(\hat{\psi} \circ \omega^{\#}\right)(l)-\frac{1}{2}\left(\hat{\psi} \circ \omega^{\#} \circ \omega\right)(d)$. Hence, in matrix form

$$
\chi=\left[\begin{array}{ccc}
\psi & 0 & 0 \\
\omega & \text { Id } & 0 \\
-\frac{1}{2} \hat{\psi} \circ \omega^{\#} \circ \omega-\hat{\psi} \circ \omega^{\#} & \hat{\psi}
\end{array}\right] .
$$

Let 


$$
\nu=\left[\begin{array}{ccc}
0 & 0 & 0 \\
\omega & 0 & 0 \\
0 & -\omega^{\#} & 0
\end{array}\right]
$$

so $\nu: E \rightarrow E$. The definition of $\omega^{\#}$ shows that $\nu$ is a skew transformation of $E$. Thus, $\exp (\nu)$ is an isometry of $E$. We can write

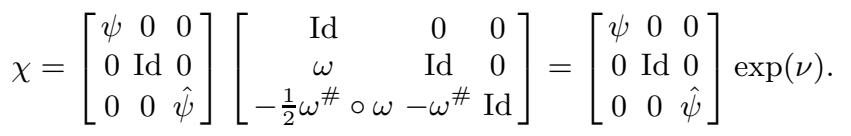

The first factor is an isometry $E \rightarrow E^{(s)}$ by the definition of $\hat{\psi}$. Thus, $\chi: E \rightarrow E^{(s)}$ is an isometry.

Since $\psi$ and $\omega$ are $\Lambda$-graded, so are $\hat{\psi}, \omega^{\#}, \eta$ and $\chi$. Also, $\omega\left(D_{0}\right) \subseteq \mathfrak{h}$, so $\chi(H) \subseteq$ $\psi\left(D_{0}\right) \oplus\left(\omega\left(D_{0}\right)+\mathfrak{h}\right) \oplus \eta\left(E_{0}\right) \subseteq H^{(s)}$. But since $D_{0}^{(s)}=\psi\left(D_{0}\right)$ and $C_{0}^{(s)}=\hat{\psi}\left(C_{0}\right)$, we have $\operatorname{dim}\left(H^{(s)}\right)=\operatorname{dim}(H)$, so $\chi(H)=H^{(s)}$.

It remains to show that $\chi$ is a homomorphism of Lie algebras. Let $\sigma^{\prime}=$ $\rho^{-1}\left(\sigma_{D^{(s)}}\right) \in Z^{2}(M, C)$. Then, $\sigma_{D^{(s)}}\left(\xi\left(m_{1}\right), \xi\left(m_{2}\right)\right)=\hat{\psi}\left(\sigma^{\prime}\left(m_{1}, m_{2}\right)\right)$ for $m_{1}, m_{2} \in$ $M$, so

$$
\sigma^{\prime}\left(m_{1}, m_{2}\right)(d)=\sigma_{D^{(s)}}\left(\xi\left(m_{1}\right), \xi\left(m_{2}\right)\right)(\psi(d))
$$

for $d \in D$. We will show that $\sigma^{\prime}=\sigma_{D}+\delta(\kappa)$ where $\kappa: M \rightarrow C$ is the 1 -cocycle

$$
\kappa(d+l)=\frac{1}{2} \omega^{\#}(\omega(d))+\omega^{\#}(l)
$$

and $\delta(\kappa)$ is the coboundary of $\kappa$; i.e.,

$$
\delta(\kappa)\left(m_{1}, m_{2}\right)=m_{2} \cdot \kappa\left(m_{1}\right)-m_{1} \cdot \kappa\left(m_{2}\right)-\kappa\left(\left[m_{1}, m_{2}\right]\right) .
$$

Indeed, using linearity and skew symmetry, it suffices to check this for the pairs $\left(l_{1}, l_{2}\right),\left(l_{1}, d_{2}\right)$, and $\left(d_{1}, d_{2}\right)$. For this recall that $L \cdot C=0,[\omega(D), \omega(D)] \subseteq$ $[\operatorname{Cent}(L) \mathfrak{h}, \operatorname{Cent}(L) \mathfrak{h}]=0$, and that $\omega$ is a derivation of $D$ into $L$. We have

$$
\begin{aligned}
\sigma^{\prime}\left(l_{1}, l_{2}\right)(d) & =\sigma_{D^{(s)}}\left(l_{1}, l_{2}\right)(\psi(d))=\left(\psi(d)\left(l_{1}\right) \mid l_{2}\right) \\
& =\left(\left(d-\operatorname{ad}_{L}(\omega(d))\left(l_{1}\right) \mid l_{2}\right)=\left(d\left(l_{1}\right)-\left[\omega(d), l_{1}\right] \mid l_{2}\right)\right. \\
& =\left(d\left(l_{1}\right) \mid l_{2}\right)-\left(\omega(d) \mid\left[l_{1}, l_{2}\right]\right)=\left(\sigma_{D}\left(l_{1}, l_{2}\right)-\omega^{\#}\left(\left[l_{1}, l_{2}\right]\right)\right)(d),
\end{aligned}
$$

while

$$
\left.\delta(\kappa)\left(l_{1}, l_{2}\right)=l_{1} \cdot \kappa\left(l_{2}\right)-l_{2} \cdot \kappa\left(l_{1}\right)-\kappa\left(\left[l_{1}, l_{2}\right]\right)=-\kappa\left(\left[l_{1}, l_{2}\right]\right)=-\omega^{\#}\left(\left[l_{1}, l_{2}\right]\right)\right) .
$$

Also,

$$
\begin{aligned}
\sigma^{\prime}\left(d_{1}, d_{2}\right)(d) & =\sigma_{D^{(s)}}\left(\psi\left(d_{1}\right)+\omega\left(d_{1}\right), \psi\left(d_{2}\right)+\omega\left(d_{2}\right)\right)(\psi(d)) \\
& =\left(\psi(d)\left(\omega\left(d_{1}\right)\right) \mid \omega\left(d_{2}\right)\right) \\
& =\left(\left(d-\operatorname{ad}_{L}(\omega(d))\left(\omega\left(d_{1}\right)\right) \mid \omega\left(d_{2}\right)\right)\right. \\
& =\left(d\left(\omega\left(d_{1}\right)\right) \mid \omega\left(d_{2}\right)\right)
\end{aligned}
$$

and 


$$
\begin{aligned}
2(\delta(\kappa) & \left.\left(d_{1}, d_{2}\right)\right)(d)=\left(d_{1} \cdot \omega^{\#}\left(\omega\left(d_{2}\right)\right)-d_{2} \cdot \omega^{\#}\left(\omega\left(d_{1}\right)\right)-\omega^{\#}\left(\omega\left(\left[d_{1}, d_{2}\right]\right)\right)\right)(d) \\
= & \left.-\left(\omega\left(d_{2}\right) \mid \omega\left(\left[d_{1}, d\right]\right)\right)+\left(\omega\left(d_{1}\right) \mid \omega\left(\left[d_{2}, d\right]\right)\right)-\left(\omega\left(\left[d_{1}, d_{2}\right]\right)\right) \mid \omega(d)\right) \\
= & -\left(\omega\left(d_{2}\right) \mid d_{1}(\omega(d))-d\left(\omega\left(d_{1}\right)\right)\right)+\left(\omega\left(d_{1}\right) \mid d_{2}(\omega(d))-d\left(\omega\left(d_{2}\right)\right)\right) \\
& \quad-\left(d_{1}\left(\omega\left(d_{2}\right)\right)-d_{2}\left(\omega\left(d_{1}\right)\right) \mid \omega(d)\right) \\
& =\left(\omega\left(d_{2}\right) \mid d\left(\omega\left(d_{1}\right)\right)\right)-\left(\omega\left(d_{1}\right) \mid d\left(\omega\left(d_{2}\right)\right)\right) \\
& =2\left(d\left(\omega\left(d_{1}\right)\right) \mid \omega\left(d_{2}\right)\right) .
\end{aligned}
$$

Finally,

$$
\sigma^{\prime}\left(d_{1}, l_{2}\right)(d)=\sigma_{D^{(s)}}\left(\psi\left(d_{1}\right)+\omega\left(d_{1}\right), l_{2}\right)(d)=\left(d \cdot \omega\left(d_{1}\right), l_{2}\right)
$$

and

$$
\begin{aligned}
\left(\delta(\kappa)\left(d_{1}, l_{2}\right)\right)(d) & =\left(d_{1} \cdot \omega^{\#}\left(l_{2}\right)-l_{2} \cdot \frac{1}{2} \omega^{\#}\left(\omega\left(d_{1}\right)\right)-\omega^{\#}\left(\left[d_{1}, l_{2}\right]\right)\right)(d) \\
& =-\left(l_{2} \mid \omega\left(\left[d_{1}, d\right]\right)\right)+\left(l_{2} \mid d_{1} \cdot \omega(d)\right) \\
& =\left(l_{2} \mid d \cdot \omega\left(d_{1}\right)\right) .
\end{aligned}
$$

So $\sigma^{\prime}=\sigma_{D}+\delta(\kappa)$ as claimed.

Since $\sigma^{\prime}+\tau=\sigma_{D}+\tau+\delta(\kappa)$, the map $m+f \rightarrow m+f-\kappa(m)$ for $m \in M$, $f \in C$ is a Lie algebra isomorphism of $E=E(L, D, \tau)$ with $\operatorname{Ext}\left(M, C, \sigma^{\prime}+\tau\right)$. We can write this isomorphism as

$$
\left[\begin{array}{ccc}
\mathrm{Id} & 0 & 0 \\
0 & \mathrm{Id} & 0 \\
-\frac{1}{2} \omega^{\#} \circ \omega & -\omega^{\#} & \mathrm{Id}
\end{array}\right]:\left[\begin{array}{c}
D \\
L \\
C
\end{array}\right] \rightarrow\left[\begin{array}{c}
D \\
L \\
C
\end{array}\right]
$$

Since $\sigma_{D^{(s)}}+\tau^{(s)}=\rho\left(\sigma^{\prime}+\tau\right)$, we also have that

$$
\xi \oplus \hat{\psi}=\left[\begin{array}{ccc}
\psi & 0 & 0 \\
\omega & \operatorname{Id} & 0 \\
0 & 0 & \hat{\psi}
\end{array}\right]
$$

is a Lie algebra isomorphism of $\operatorname{Ext}\left(M, C, \sigma^{\prime}+\tau\right)$ with $E^{(s)}$. Thus,

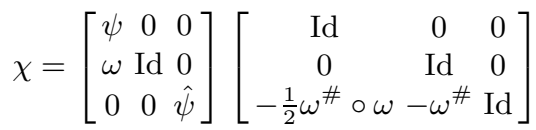

is a Lie algebra isomorphism from $E$ to $E^{(s)}$.

Corollary 6.3. Let $L$ be a centreless Lie $\Lambda$-torus of type $\Delta$, and let $L^{\prime}$ be a centreless Lie $\Lambda^{\prime}$-torus of type $\Delta^{\prime}$. Suppose that $L$ is isotopic to $L^{\prime}$. Then, there is a bijection $(D, \tau) \mapsto\left(D^{\prime}, \tau^{\prime}\right)$ from $\mathcal{P}(L)$ onto $\mathcal{P}\left(L^{\prime}\right)$ such that $E(L, D, \tau)$ is isomorphic as an EALA to $E\left(L^{\prime}, D^{\prime}, \tau^{\prime}\right)$ for all $(D, \tau) \in \mathcal{P}(L)$.

Proof. If $L$ and $L$ are bi-isomorphic, the result is clear. So we can assume that $L^{\prime}=$ $L^{(s)}$ is an isotope of $L$. Define $T: \mathcal{P}(L) \rightarrow \mathcal{P}\left(L^{\prime}\right)$ by $T(\mathcal{D}, \tau)=\left(\mathcal{D}^{(s)}, \tau^{(s)}\right)$ using the notation of Theorem 6.2. Similarly, we have a map $T^{\prime}: \mathcal{P}\left(L^{\prime}\right) \rightarrow \mathcal{P}\left(L^{\prime(-s)}\right)=\mathcal{P}(L)$, and it is clear that $T$ and $T^{\prime}$ are inverses of one another. So $T$ is a bijection. 
Less precisely (but more succinctly), Corollary 6.3 says that if the centerless Lie tori $L$ and $L^{\prime}$ are isotopic then the families $\{E(L, D, \tau)\}_{(D, \tau) \in \mathcal{P}(L)}$ and $\left\{E\left(L^{\prime}, D^{\prime}, \tau^{\prime}\right)\right\}_{\left(D^{\prime}, \tau^{\prime}\right) \in \mathcal{P}\left(L^{\prime}\right)}$ of EALAs are bijectively isomorphic. Using this language, Theorem 4.2, Theorem 6.1 and Corollary 6.3 together tell us that Construction 4.1 provides a one-to-one correspondence between centreless Lie tori up to isotopy and families of EALAs up to bijective isomorphism.

Remark 6.4. Suppose that $L$ is a centreless Lie torus. To construct all of the members of the family $\{E(L, D, \tau)\}_{(D, \tau) \in \mathcal{P}(L)}$ of EALAs corresponding to $L$, one must do the following: (i) Calculate the centroid $\operatorname{Cent}(L)$ and identify it explicitly with $\mathbb{F}[\Gamma]$ (in which case $\operatorname{SCDer}(L)$ is completely understood from (3)); (ii) Determine the $\Gamma$-graded subalgebras $D$ of $\operatorname{SCDer}(L)$ which satisfy (4.1a); (iii) For each $D$ in (ii), determine all 2-cocycles of $D$ satisfying (4.1b). We note that (i) can be carried out for each type $\Delta$ using the coordinatization theorems (see $\S 7$ below) and the results and methods in [BN, §5]. However, finding a general approach to (ii) and (iii) seems to be much more difficult. (See [BiN, p.3] for some remarks on problem (iii) and see [BGK, Remark 3.71(b)] for an example of a nontrivial choice of $\tau$.) We do however note that the tasks (ii) and (iii) are independent of the type $\Delta$; in fact they depend only on the rank of $\Gamma$.

\section{Coordinatization of Lie tori}

In the classical study of finite dimensional split simple Lie algebras, one can construct the algebras using special linear, orthogonal and symplectic matrix constructions along with some exceptional constructions starting from Jordan algebras and alternative algebras. (See for example [S, §III.8, IV.3 and IV.4].)

Similar coordinatization theorems have been proved for centreless Lie tori. Very roughly speaking these results show that a centerless Lie torus of a given type can be constructed as a "matrix algebra" over a "coordinate torus", which for some types is nonassociative. References for each type are: $\mathrm{A}_{1}$ : [Y1]; $\mathrm{A}_{2}$ : $[\mathrm{BGKN}, \mathrm{Y} 4] ; \mathrm{A}_{l}(l \geq 3)$, $D_{l}(l \geq 4), E_{6}, E_{7}$ and $E_{8}:[\mathrm{BGK}, \mathrm{Y} 2] ; \mathrm{B}_{2}=\mathrm{C}_{2}$ : [AG, BY]; $\mathrm{B}_{l}(l \geq 3):[\mathrm{AG}, \mathrm{Y} 5, \mathrm{AB}] ;$ $\mathrm{C}_{l}(l \geq 3):[\mathrm{AG}, \mathrm{BY}] ; \mathrm{F}_{4}$ and $\mathrm{G}_{2}:[\mathrm{AG}] ; \mathrm{BC}_{1}[\mathrm{AFY}] ; \mathrm{BC}_{2}:[\mathrm{F} 1]$; and finally $\mathrm{BC}_{l}(l \geq 3)$ : $[\mathrm{AB}]$. We note that these theorems were in some cases proved before the notion of Lie torus had been introduced and were instead formulated in the language of EALAs. Also, in some cases the theorems were proved over the complex field. Nevertheless the translation to the language of Lie tori and the extension to arbitrary base fields of characteristic 0 are not difficult.

In the following sections, we recall the coordinatization theorems for types $\mathrm{A}_{1}$, $\mathrm{A}_{2}, \mathrm{~A}_{l}(l \geq 3)$ and $\mathrm{C}_{l}(l \geq 4)$, which use respectively Jordan tori, alternative tori, associative tori and associative tori with involution as coordinates. First we recall the definitions of these types of tori.

Recall that a Jordan algebra is an algebra with commutative product satisfying $a^{2}(a b)=a\left(a^{2} b\right)$, and an alternative algebra is an algebra with product satisfying $a(a b)=a^{2} b$ and $(b a) a=b a^{2}$ (see for example [S] or [Mc2]).

Definition 7.1. Suppose that $A=\bigoplus_{\lambda \in \Lambda} A^{\lambda}$ is a $\Lambda$-graded Jordan algebra, alternative algebra or associative algebra. A is said to be a Jordan, alternative or associative $\Lambda$-torus respectively if: 
(i) For $\lambda \in \operatorname{supp}_{\Lambda}(A), A^{\lambda}$ is spanned by a single invertible element of $A .^{10}$

(ii) $\Lambda$ is generated as a group by $\operatorname{supp}_{\Lambda}(A)$.

Note of course that any associative torus is an alternative torus. An associative 1 torus with involution is a pair $(A, \iota)$, where $A$ is an associative $\Lambda$-torus and $\iota$ is a graded involution of $A$.

Remark 7.2. Suppose that $A$ is a Jordan or alternative $\Lambda$-torus, and let $S=$ $\operatorname{supp}_{\Lambda}(A)$. Then, it is clear that $-S=S$. Moveover, if $A$ is alternative, it is easy to see that $S$ is closed under addition, so $S=\Lambda$. However, this is not true in the Jordan case, although $S$ is closed under the operation $(\lambda, \mu) \mapsto \lambda+2 \mu$.

Example 7.3. Let $\mathbf{q}=\left(q_{i j}\right)$ be an $n \times n$-matrix over $\mathbb{F}$ with $q_{i i}=1$ and $q_{i j}=q_{j i}^{-1}$ for all $i, j$. Let $\mathbb{F}_{\mathbf{q}}=\mathbb{F}_{\mathbf{q}}\left[x_{1}^{ \pm 1}, \ldots, x_{n}^{ \pm 1}\right]$ be the associative algebra over $\mathbb{F}$ presented by the generators $x_{i}, x_{i}^{-1}, i=1, \ldots, n$, subject to the relations

$$
x_{i} x_{i}^{-1}=x_{i}^{-1} x_{i}=1 \quad \text { and } \quad x_{j} x_{i}=q_{i j} x_{i} x_{j}, \quad 1 \leq i, j \leq n .
$$

Then, $\mathbb{F}_{\mathbf{q}}$ has a natural $\mathbb{Z}^{n}$-grading with $\left(\mathbb{F}_{\mathbf{q}}\right)^{\left(l_{1}, \ldots, l_{n}\right)}=\mathbb{F} x_{1}^{l_{1}} \ldots x_{n}^{l_{n}}$. The $\mathbb{Z}^{n}$-graded algebra $\mathbb{F}_{\mathbf{q}}$ is an associative $\mathbb{Z}^{n}$-torus called the quantum torus determined by $\mathbf{q}^{11}$ Conversely, it is easy to see that any associative torus is isograded-isomorphic to a quantum torus. Recently, rational quantum tori, that is quantum tori $\mathbb{F}_{\mathbf{q}}$ with each $q_{i j}$ a root of unity, have been classified up to isograded-isomorphism by K.-H. Neeb in [Ne, Thm. III.4] as tensor products of rational quantum $\mathbb{Z}^{2}$-tori and an algebra of Laurent polynomials. (In [H, Thm. 4.8], J.T. Hartwig gave a tensor product decomposition of rational quantum tori but he did not give a condition for isomorphism.)

In the following sections, we will give some further examples of the tori defined in Definition 7.1.

\section{Type $A_{1}$}

In this section we assume that

$$
\Delta=\{\alpha, 0,-\alpha\}
$$

is the root system of type $\mathrm{A}_{1}$, and $Q=Q(\Delta)=\mathbb{Z} \alpha$.

To construct a centreless Lie $\Lambda$-torus of type $\Delta$, we use the Tits-Kantor-Koecher construction. (See [Mc2, p.13] for the facts mentioned below about this construction.) We begin with a Jordan $\Lambda$-torus $A$ and we define operators $V_{x, y} \in \operatorname{End}(A)$ for $x, y \in A$ by $V_{x, y} z=\{x, y, z\}:=2((x y) z+(z y) x-(z x) y)$. These operators satisfy the identity $\left[V_{x, y}, V_{z, w}\right]=V_{\{x, y, z\}, w}-V_{z,\{y, x, w\}}$, so their span $V_{A, A}$ is a Lie algebra under the commutator product. Moreover, the map $*$ defined by $V_{x, y} \mapsto-V_{y, x}$ is a well-defined automorphism of $V_{A, A}$. We now set

$$
L(A)=\operatorname{TKK}(A):=A_{1} \oplus V_{A, A} \oplus A_{-1},
$$

${ }^{10}$ We are using the standard definitions of invertibility for Jordan algebras [Mc2, p.210], alternative algebras [S, p.38] and associative algebras.

${ }^{11}$ If $n=0$, we interpret $\mathbf{q}=\emptyset$ and $\mathbb{F}_{\mathbf{q}}=\mathbb{F}$. 
where, for $i= \pm 1, A_{i}$ is a copy of the vector space $A$ under the linear bijection $x \mapsto x_{i}$. Then $L(A)$ is a Lie algebra, called the Tits-Kantor-Koecher Lie algebra of $A$, under the product defined by:

$$
\begin{gathered}
{\left[T, x_{1}\right]=(T x)_{1}, \quad\left[T, y_{-1}\right]=\left(T^{*} y\right)_{-1}, \quad\left[x_{i}, y_{i}\right]=0(i= \pm 1),} \\
{\left[x_{1}, y_{-1}\right]=V_{x, y}, \quad\left[T, T^{\prime}\right]=T T^{\prime}-T^{\prime} T,}
\end{gathered}
$$

for $x, y \in A, T, T^{\prime} \in V_{A, A}$. Finally, $L(A)$ is a $Q \times \Lambda$-graded algebra with

$$
L(A)_{\alpha}^{\lambda}=\left(A^{\lambda}\right)_{1}, \quad L(A)_{0}^{\lambda}=\sum_{\mu+\nu=\lambda} V_{A^{\mu}, A^{\nu}}, \quad L(A)_{-\alpha}^{\lambda}=\left(A^{\lambda}\right)_{-1},
$$

for $\lambda \in \Lambda$ and $L(A)_{\beta}^{\lambda}=0$ for $\lambda \in \Lambda, \beta \in Q \backslash \Delta$. Yoshii has shown that $L(A)$ is a centreless Lie $\Lambda$-torus of type $\Delta$, and he proved the following coordinatization theorem.

Theorem 8.1 ([Y1, Thm. 1]). Any centreless Lie $\Lambda$-torus of type $\Delta$ is gradedisomorphic to the Lie $\Lambda$-torus $L(A)=\operatorname{TKK}(A)$ constructed from a Jordan $\Lambda$ torus $A$.

Yoshii went on to describe five families of Jordan $\mathbb{Z}^{n}$-tori and then he showed that every Jordan $\Lambda$-torus with $\operatorname{rank}(\Lambda)=n$ is isograded-isomorphic to a torus in one of the families [Y1, Thm. 2]. The simplest of these families consists of the tori $\mathbb{F}_{\mathbf{q}}^{+}$, where $\mathbb{F}_{\mathbf{q}}^{+}$denotes the $\mathbb{Z}^{n}$-graded algebra $\mathbb{F}_{\mathbf{q}}$ with multiplication $x \cdot y=\frac{1}{2}(x y+y x)$.

Definition 8.2 (Isotopes of Jordan tori). Let $A$ be Jordan $\Lambda$-torus and let $u$ be a nonzero homogeneous element of $A$. So $u \in A^{-\rho}$, where $\rho \in \operatorname{supp}_{\lambda}(A)$. Let $A^{(u)}$ be the algebra with underlying vector space $A$ and product $\cdot_{u}$ defined $b y$

$$
x \cdot u=\frac{1}{2}\{x, u, y\}
$$

Then, since $u$ is invertible in $A, A^{(u)}$ is a Jordan algebra and the identity element of $A^{(u)}$ is $1^{(u)}=u^{-1}$ [Mc2, p.86]. So $1^{(u)} \in A^{\rho}$. We endow $A^{(u)}$ with a $\Lambda$-grading by setting

$$
\left(A^{(u)}\right)^{\lambda}=A^{\lambda+\rho}
$$

for $\lambda \in \Lambda$. It is easily checked that $A^{(u)}$ is a Jordan $\Lambda$-torus which we call the $u$ isotope of the Jordan torus $A$. It is also easily checked that up to graded-isomorphism $A^{(u)}$ does not depend on the choice of nonzero $u$ in $A^{-\rho}$.

Suppose that $u, v$ are nonzero homogeneous elements of a Jordan $\Lambda$-torus. It is well known that

$$
A^{(1)}=A, \quad\left(A^{(u)}\right)^{(v)}=A^{\left(U_{u} v\right)} \quad \text { and } \quad\left(A^{(u)}\right)^{\left(u^{-2}\right)}=A,
$$

as algebras [Mc2, Proposition 7.2.1], where $U_{u} v=\frac{1}{2}\{u, v, u\}$. It is easy to check that these are also equalities of $\Lambda$-graded Jordan algebras.

Definition 8.3. Suppose that $A$ is a Jordan $\Lambda$-torus and $A^{\prime}$ is a Jordan $\Lambda^{\prime}$-torus. We say that $A$ is isotopic to $A^{\prime}$ if some isotope of $A$ is isograded-isomorphic to $A^{\prime}$. In that case, we write $A \sim A^{\prime}$. It follows from (26) that $\sim$ is an equivalence relation on the class of Jordan tori. 
The following lemma is our key to understanding the connection between isotopy for Lie tori and isotopy for Jordan tori. In fact its proof (see (27) and (28) below) shows that the definition of isotope for Jordan tori is determined without foreknowledge from the definition of isotope for Lie tori. We will see the same phenomenon for alternative tori in $\S 9$ and for associative tori with involution in $\S 11$.

Lemma 8.4 (Key lemma for type $\mathbf{A}_{1}$ ). Suppose that $A^{\prime}$ is a Jordan $\Lambda^{\prime}$-torus, $A$ is an Jordan $\Lambda$-torus, and $s \in \operatorname{Hom}(Q, \Lambda)$ is admissible for $L(A)$. Suppose that $\varphi: L\left(A^{\prime}\right) \rightarrow L(A)^{(s)}$ is a bi-isomorphism with $\varphi_{r}=1$. Then, $-s(\alpha) \in \operatorname{supp}_{\Lambda}(A)$ and for any nonzero $u$ in $A^{-s(\alpha)}$ there is an isograded-isomorphism $\eta: A^{\prime} \rightarrow A^{(u)}$ such that $\eta_{g r}=\varphi_{e}$.

Proof. Note first that since $s$ is admissible, we have $s(\alpha) \in \Lambda_{\alpha}(L(A))=\operatorname{supp}_{\Lambda}(A)$, so $-s(\alpha) \in \operatorname{supp}_{\Lambda}(A)$. Thus we can choose nonzero $u$ in $A^{-s(\alpha)}$ as suggested.

Let $1^{\prime}$ denote the identity in $A^{\prime}$. Then

$$
\varphi\left(1_{-1}^{\prime}\right) \in \varphi\left(L\left(A^{\prime}\right)_{-\alpha}^{0}\right)=\left(\left(L(A)^{(s)}\right)_{-\alpha}^{0}=L(A)_{-\alpha}^{-s(\alpha)}=\left(A^{-s(\alpha)}\right)_{-1}\right.
$$

So replacing $u$ by a scalar multiple we can assume that $\varphi\left(1_{-1}^{\prime}\right)=u_{-1}$. Now define a linear bijection $\eta: A^{\prime} \rightarrow A$ by

$$
(\eta x)_{1}=\varphi\left(x_{1}\right)
$$

for $x \in A^{\prime}$. Then, for $x, y \in A^{\prime}$, we have

$$
\begin{aligned}
(\eta(x y))_{1} & =\varphi\left((x y)_{1}\right)=\frac{1}{2} \varphi\left(\left\{x, 1^{\prime}, y\right\}\right)=\frac{1}{2} \varphi\left(\left[\left[x_{1}, 1_{-1}^{\prime}\right], y_{1}\right]\right) \\
& =\frac{1}{2}\left[\left[(\eta x)_{1}, u_{-1}\right],(\eta y)_{1}\right]=\left(\frac{1}{2}\{\eta x, u, \eta y\}\right)_{1}=(\eta(x) \cdot u \eta(y))_{1},
\end{aligned}
$$

and, for $\lambda \in \Lambda^{\prime}$,

$$
\begin{aligned}
\left(\eta\left(A^{\prime \lambda}\right)\right)_{1} & =\varphi\left(\left(A^{\prime \lambda}\right)_{1}\right)=\varphi\left(L\left(A^{\prime}\right)_{\alpha}^{\lambda}\right)=\left(L^{(s)}\right)_{\alpha}^{\varphi_{e}(\lambda)} \\
& =\left(A^{\varphi_{e}(\lambda)+s(\alpha)}\right)_{1}=\left(\left(A^{(u)}\right)^{\varphi_{e}(\lambda)}\right)_{1} .
\end{aligned}
$$

Proposition 8.5. Let $A$ be a Jordan $\Lambda$-torus, and suppose that $s \in \operatorname{Hom}(Q, \Lambda)$ is admissible for $L(A)$. Then, $-s(\alpha) \in \operatorname{supp}_{\Lambda}(A)$ and for any nonzero $u$ in $A^{-s(\alpha)}$, we have $L(A)^{(s)} \simeq_{Q \times \Lambda} L\left(A^{(u)}\right)$.

Proof. By Theorem 8.1, we have $L(A)^{(s)} \simeq_{Q \times \Lambda} L\left(A^{\prime}\right)$ for some Jordan $\Lambda$-torus $A^{\prime}$. So we have a bi-isomorphism $\varphi: L\left(A^{\prime}\right) \rightarrow L(A)^{(s)}$ with $\varphi_{r}=1$ and $\varphi_{e}=1$. Lemma 8.4 now tells us that $A^{\prime} \simeq_{\Lambda} A^{(u)}$, so $L\left(A^{\prime}\right) \simeq_{Q \times \Lambda} L\left(A^{(u)}\right)$. Hence $L(A)^{(s)} \simeq_{Q \times \Lambda}$ $L\left(A^{(u)}\right)$.

Our main theorem in this section, gives necessary and sufficient conditions for two centreless Lie tori $L(A)$ and $L\left(A^{\prime}\right)$ to be isotopic. To do this, we need to first give necessary and sufficient conditions for these Lie tori to be bi-isomorphic.

Theorem 8.6. Suppose that $A$ is a Jordan $\Lambda$-torus and $A^{\prime}$ is a Jordan $\Lambda^{\prime}$-torus. Let $L(A)=\operatorname{TKK}(A)$ and $L\left(A^{\prime}\right)=\operatorname{TKK}\left(A^{\prime}\right)$. Then 
(i) $L(A) \simeq_{b i} L\left(A^{\prime}\right) \Longleftrightarrow A \simeq_{i g} A^{\prime}$.

(ii) $L(A) \sim L\left(A^{\prime}\right) \Longleftrightarrow A \sim A^{\prime}$.

Proof. Let $L=L(A)$ and $L^{\prime}=L\left(A^{\prime}\right)$.

(i): The proof of " $\Leftarrow$ " is clear, so we prove " $\Rightarrow$ ". Suppose $\varphi: L^{\prime} \rightarrow L$ is a biisomorphism. Then $\varphi_{r}= \pm 1$, so $\varphi_{r}$ is in the Weyl group of $\Delta$. Therefore, by Lemma 3.8 , we can assume that $\varphi_{r}=1$. Thus, by Lemma 8.4 with $s=0$ and $u=1$, we have $A^{\prime} \simeq_{i g} A$.

(ii): " $\Rightarrow$ " By assumption, $L^{\prime} \simeq_{b i} L^{(s)}$ for some $s \in \operatorname{Hom}(Q, \Lambda)$ that is admissible for $L$. So, choosing $u$ as in Proposition 8.5, we have $L^{\prime} \simeq_{b i} L\left(A^{(u)}\right)$. By $(\mathrm{i}), A^{\prime} \simeq_{i g}$ $A^{(u)}$.

" $\Leftarrow$ " By (i) we can assume that $A^{\prime}=A^{(u)}$, where $u$ is a nonzero homogeneous element of $A$. Then $u \in A^{-\rho}$, where $\rho \in \operatorname{supp}_{\Lambda} L=\Lambda_{\alpha}(L)$. So $s: Q \rightarrow \Lambda$ defined by $s(\alpha)=\rho$ is admissible for $L$, and hence $L^{\prime} \simeq_{b i} L^{(s)}$ by Proposition 8.5.

We will next give an example of two centreless Lie tori that are isotopic but not bi-isomorphic. For this we need a little preparation.

Remark 8.7. Suppose that $A$ is a Jordan $\Lambda$-torus, and let $\operatorname{Cent}(A)$ be the centroid of $A$. It is clear that $\operatorname{Cent}(A)$ is $\Lambda$-graded and that the support $\Gamma=\Gamma(A)$ of $\operatorname{Cent}(A)$ is a subgroup of $\Lambda[\mathrm{Y} 1, \S 3]$. Also, if $S=\operatorname{supp}_{\Lambda}(A)$, it is clear that $S$ is a union of cosets of $\Gamma$ in $\Lambda$, and we let $S / \Gamma=\{\lambda+\Gamma \mid \lambda \in S\} \subseteq \Lambda / \Gamma$. If $S / \Gamma$ is finite, we denote the sum of the elements of $S / \Gamma$ in $\Lambda / \Gamma$ by $\Sigma(S / \Gamma)$.

Lemma 8.8. Suppose $A$ is a Jordan $\Lambda$-torus with $S / \Gamma$ finite and $\Sigma(S / \Gamma)=0$, where $\Gamma=\Gamma(A)$ and $S=\operatorname{supp}_{\Lambda}(A)$. If $\rho \in S$ with $|S / \Gamma| \rho \notin \Gamma$ and $0 \neq u \in A^{-\rho}$, then $A^{(u)} \not_{i g} A$.

Proof. Let $\Gamma^{(u)}=\Gamma\left(A^{(u)}\right)$ and $S^{(u)}=\operatorname{supp}_{\Lambda}\left(A^{(u)}\right)$. It is easily checked that $\operatorname{Cent}\left(A^{(u)}\right)=\operatorname{Cent}(A)$, so $\Gamma^{(u)}=\Gamma$. Also, $S^{(u)}=S-\rho$, so $\Sigma\left(S^{(u)} / \Gamma\right)=$ $\Sigma(S / \Gamma)-|S / \Gamma|(\rho+\Gamma)=-|S / \Gamma|(\rho+\Gamma) \neq 0$. But then $\Sigma(S / \Gamma)=0$ and $\Sigma\left(S^{(u)} / \Gamma\right) \neq 0$ imply our conclusion.

Our example uses Jordan tori of Clifford type [Y1, Example 5.2] as coordinate algebras.

Example 8.9. Let $\Lambda=\mathbb{Z}^{3}$ and let $R=\mathbb{F}[2 \Lambda]$ be the group algebra of $2 \Lambda$ with its natural grading by $2 \Lambda$ (and hence by $\Lambda$ ). Let $\left\{\lambda_{1}, \lambda_{2}, \lambda_{3}\right\}$ be the standard basis of $\Lambda$ and let $\lambda_{4}=\lambda_{1}+\lambda_{2}+\lambda_{3}$. Let $V=\bigoplus_{i=1}^{4} R x_{i}$ be the free $R$-module with base $\left\{x_{1}, x_{2}, x_{3}, x_{4}\right\}$, and give $V$ the $\Lambda$-grading such that $\operatorname{deg}\left(t^{\mu} x_{i}\right)=\mu+\lambda_{i}$ for $\mu \in 2 \Lambda$ and $1 \leq i \leq 4$. Define a $\Lambda$-graded $R$-bilinear form $f: V \times V \rightarrow R$ by $f\left(x_{i}, x_{j}\right)=\delta_{i, j} t^{2} \lambda_{i}$ for $1 \leq i, j \leq 4$. Finally, let $A=R \oplus V$ with the direct sum $\Lambda$-grading, and define a product in $A$ by

$$
(r+v)\left(r^{\prime}+v^{\prime}\right)=r r^{\prime}+f\left(v, v^{\prime}\right)+r v^{\prime}+r^{\prime} v
$$

for $r, r^{\prime} \in R, v, v^{\prime} \in V$. Then $A$ is a Jordan torus. (The algebra $A$ is called a spin factor in [Mc2, p.74].) Further, using the notation of Lemma 8.8, $\Gamma=2 \Lambda$ and $S=(2 \Lambda) \cup\left(\cup_{i=1}^{4}\left(2 \Lambda+\lambda_{i}\right)\right)$, so $S / \Gamma$ is finite and $\Sigma(S / \Gamma)=0$. Also $|S / \Gamma| \rho=5 \rho \notin \Gamma$ for $\rho \in S \backslash \Gamma$. So for any nonzero homogeneous element $u$ in $A \backslash R$, we have, by Lemma 8.8, that $A \neq_{i g} A^{(u)}$. Therefore, for any such $u$, we see by Theorem 8.6 that $L(A) \not_{b i} L\left(A^{(u)}\right)$ but $L(A) \sim L\left(A^{(u)}\right)$. 


\section{Type $\mathrm{A}_{2}$}

Suppose in this section that

$$
\Delta=\{0\} \cup\left\{\varepsilon_{i}-\varepsilon_{j} \mid 1 \leq i \neq j \leq 3\right\}
$$

is the root system of type $\mathrm{A}_{2}$ (where $\varepsilon_{1}, \varepsilon_{2}, \varepsilon_{3}$ is a basis for a space containing $\Delta$ ), and $Q=Q(\Delta)$. We let $\alpha_{1}=\varepsilon_{1}-\varepsilon_{2}$ and $\alpha_{2}=\varepsilon_{2}-\varepsilon_{3}$, in which case $\left\{\alpha_{1}, \alpha_{2}\right\}$ is a base for the root system $\Delta$.

Let $A$ be an alternative $\Lambda$-torus. We construct a centreless Lie $\Lambda$-torus of type $\Delta$ from $A$ using J. Faulkner's $3 \times 3$-projective elementary construction. (See [F1, Appendix, (A6)] for the properties mentioned below about this construction. Note however that we are using the notation $e_{i j}(x)$ in place of the notation $v_{i j}(x)$ that is used in [F1].) Recall that the $3 \times 3$ projective elementary Lie algebra is the Lie algebra

$$
L(A)=\operatorname{pe}_{3}(A):=L_{0} \oplus\left(\bigoplus_{1 \leq i \neq j \leq 3} e_{i j}(A)\right),
$$

where $L_{0}$ and $e_{i j}(A)$ are subspaces of $L$ satisfying:

(a) For $i \neq j$, there is a linear bijection $x \mapsto e_{i j}(x)$ from $A$ to $e_{i j}(A)$,

(b) $\left[e_{i j}(x), e_{j k}(y)\right]=e_{i k}(x y)$ for $x, y \in A$ and distinct $i, j, k$,

(c) $\left[e_{i j}(x), e_{i j}(y)\right]=0$ for $x, y \in A, i \neq j$,

(d) $\left[L_{0}, L_{0}\right] \subseteq L_{0}$

(e) $\left[L_{0}, e_{i j}(A)\right] \subseteq e_{i j}(A)$ for $i \neq j$,

(f) $L_{0}=\sum_{1 \leq i<j \leq 3}\left[e_{i j}(A), e_{j i}(A)\right]$,

(g) $\left\{x \in L_{0}:\left[x, e_{i j}(A)\right]=0\right.$ for $\left.i \neq j\right\}=0$.

These properties characterize the Lie algebra $L(A)$ in the following sense: If $L^{\prime}(A)=$ $L_{0}^{\prime} \oplus\left(\bigoplus_{1 \leq i \neq j \leq 3} e_{i j}^{\prime}(A)\right)$ is another Lie algebra satisfying the conditions in (30), then there is a unique isomorphism from $L(A) \rightarrow L^{\prime}(A)$ such that $e_{i j}(x) \mapsto e_{i j}^{\prime}(x)$ for $i \neq j, x \in A$. Finally $L(A)$ is a $Q \times \Lambda$-graded algebra with

$$
L(A)_{\varepsilon_{i}-\varepsilon_{j}}^{\lambda}=e_{i j}\left(A^{\lambda}\right), \quad L(A)_{0}^{\lambda}=\sum_{\mu+\nu=\lambda} \sum_{1 \leq k<l \leq 3}\left[e_{k l}\left(A^{\mu}\right), e_{l k}\left(A^{\nu}\right)\right],
$$

for $i \neq j, \lambda \in \Lambda$, and $L(A)_{\alpha}^{\lambda}=0$ for $\lambda \in \Lambda, \alpha \in Q \backslash \Delta$. It is easily checked that $L(A)$ is a centreless Lie torus of type $\mathrm{A}_{2}$.

Moreover, Berman, Gao, Krylyuk and Neher (over $\mathbb{C}$ ) and Yoshii (in general) proved the following coordinatization theorem:

Theorem 9.1. ([BGKN, Lemma 3.25], [Y4, Prop. 6.3]) Any centreless Lie A-torus of type $\Delta$ is graded-isomorphic to the Lie $\Lambda$-torus $L(A)=\mathrm{pe}_{3}(A)$ constructed from an alternative $\Lambda$-torus $A .^{12}$

An important example of an alternative torus is the octonion torus. A simple way to introduce this torus uses a presentation [Y7].

$\overline{12}$ A different description of $L(A)$ was used in [BGKN] and [Y4], but it is not difficult to check that the two descriptions give Lie tori that are $Q \times \Lambda$-graded-isomorphic. 
Example 9.2. Let $\mathcal{C}(3)$ be the alternative algebra that is presented by the generators $x_{i}^{ \pm 1}, 1 \leq i \leq 3$, subject to the relations $x_{i} x_{i}^{-1}=x_{i}^{-1} x_{i}=1$ for all $i$,

$$
x_{i} x_{j}=-x_{j} x_{i} \text { for } i \neq j, \quad \text { and } \quad\left(x_{1} x_{2}\right) x_{3}=-x_{1}\left(x_{2} x_{3}\right) .
$$

Although we will not need this, one can show more concretely that each element of $\mathcal{C}(3)$ can be written uniquely as a linear combination of monomials $x^{i}=\left(x_{1}^{i_{1}} x_{2}^{i_{2}}\right) x_{3}^{i_{3}}$, $\boldsymbol{i}=\left(i_{1}, i_{2}, i_{3}\right) \in \mathbb{Z}^{3}$, with multiplication given by

$$
x^{i} x^{j}=(-1)^{\kappa(i, j)} x^{i+j},
$$

where $\kappa(\boldsymbol{i}, \boldsymbol{j})=i_{3} j_{1}+i_{2} j_{1}+i_{3} j_{2}+i_{1} j_{2} j_{3}+i_{2} j_{1} j_{3}+i_{3} j_{1} j_{2}$.

If $n \geq 3$, the graded algebra $\mathcal{C}(3) \otimes \mathbb{F}\left[x_{4}^{ \pm 1}, \ldots, x_{n}^{ \pm 1}\right]$ is an alternative $\mathbb{Z}^{n}$-torus, where $x_{1}, \ldots, x_{n}$ are assigned the degrees in the standard ordered basis for $\mathbb{Z}^{n}$ (identifying $\mathcal{C}(3)$ and $\mathbb{F}\left[x_{4}^{ \pm 1}, \ldots, x_{n}^{ \pm 1}\right]$ as subalgebras of the tensor product). The $\mathbb{Z}^{n}$-graded algebra $\mathcal{C}(3) \otimes \mathbb{F}\left[x_{4}^{ \pm 1}, \ldots, x_{n}^{ \pm 1}\right]$ is called the octonion $\mathbb{Z}^{n}$-torus.

We have the following description of alternative tori:

Theorem 9.3. ([BGKN, Thm. 1.25], [Y4, Cor. 5.13]) If $A$ is an alternative $\Lambda$-torus, then either $A$ is associative (and hence isograded-isomorphic to a quantum torus), or $n \geq 3$ and $A$ is isograded-isomorphic to the octonion $\mathbb{Z}^{n}$-torus.

Remark 9.4. Suppose $A$ is a $\Lambda$-torus. Recall that by $\operatorname{Remark} 7.2, \operatorname{supp}(A)=\Lambda$. So $\Lambda_{\alpha}(L(A))=\Lambda$ for all nonzero $\alpha \in \Delta$, and therefore any $s \in \operatorname{Hom}(Q, \Lambda)$ is admissible for $L(A)$.

Definition 9.5 (Isotopes of alternative tori). Let $A$ be an alternative 1 -torus and let $u_{1}$ and $u_{2}$ be nonzero homogeneous elements of $A$. Thus, $u_{i} \in A^{-\rho_{i}}$, where $\rho_{i} \in \Lambda$ for $i=1,2$. Let $A^{\left(u_{1}, u_{2}\right)}$ be the algebra with underlying vector space $A$ and with product $\cdot_{u_{1}, u_{2}}$ defined by:

$$
x \cdot u_{1}, u_{2} y=\left(x u_{1}\right)\left(u_{2} y\right)
$$

for $x, y \in A$. Then, since $u_{1}, u_{2}$ are invertible, $A^{\left(u_{1}, u_{2}\right)}$ is an alternative algebra with identity $1^{\left(u_{1}, u_{2}\right)}=\left(u_{1} u_{2}\right)^{-1}[M c 1$, Thm. 1 and Prop. 2]. We define a A-grading on $A^{\left(u_{1}, u_{2}\right)}$ by setting

$$
\left(A^{\left(u_{1}, u_{2}\right)}\right)^{\lambda}=A^{\lambda+\rho_{1}+\rho_{2}}
$$

for $\lambda \in \Lambda$. Then $A^{\left(u_{1}, u_{2}\right)}$ is an alternative $\Lambda$-torus which we call the $\left(u_{1}, u_{2}\right)$-isotope of $A$. It is easily checked that this torus does not depend up to graded-isomorphism on the choice of nonzero $u_{i}$ in $A^{-\rho_{i}}$.

Lemma 9.6 (Key lemma for type $\mathbf{A}_{2}$ ). Suppose that $A^{\prime}$ is an alternative $\Lambda^{\prime}$ torus, $A$ is an alternative $\Lambda$-torus, and $s \in \operatorname{Hom}(Q, \Lambda)$. Suppose that $\varphi: L\left(A^{\prime}\right) \rightarrow$ $L(A)^{(s)}$ is a bi-isomorphism with $\varphi_{r}=1$. Choose $0 \neq u_{i} \in A^{s\left(\alpha_{i}\right)}$ for $i=1,2$. Then there is an isograded-isomorphism $\eta: A^{\prime} \rightarrow A^{\left(u_{1}, u_{2}\right)}$ such that $\eta_{g r}=\varphi_{e}$.

Proof. Write $L\left(A^{\prime}\right)=L_{0}^{\prime} \oplus\left(\bigoplus_{1 \leq i \neq j \leq 3} e_{i j}^{\prime}\left(A^{\prime}\right)\right)$ as in (29). Now $\varphi\left(e_{i j}^{\prime}\left(A^{\prime \lambda}\right)\right)=$ $\varphi\left(L\left(A^{\prime}\right)_{\varepsilon_{i}-\varepsilon_{j}}^{\lambda}\right)=\left(L(A)^{(s)}\right)_{\varepsilon_{i}-\varepsilon_{j}}^{\varphi_{e}(\lambda)}=L(A)_{\varepsilon_{i}-\varepsilon_{j}}^{\varphi_{e}(\lambda)+s\left(\varepsilon_{i}-\varepsilon_{j}\right)}=e_{i j}\left(A^{\varphi_{e}(\lambda)+s\left(\varepsilon_{i}-\varepsilon_{j}\right)}\right)$ for $\lambda \in \Lambda^{\prime}, i \neq j$. Thus, for $i \neq j$, we have a linear bijection $\varphi_{i j}: A^{\prime} \rightarrow A$ with 


$$
\varphi\left(e_{i j}^{\prime}(x)\right)=e_{i j}\left(\varphi_{i j}(x)\right)
$$

for $x \in A^{\prime}$, in which case

$$
\varphi_{i j}\left(A^{\prime \lambda}\right)=A^{\varphi_{e}(\lambda)+s\left(\varepsilon_{i}-\varepsilon_{j}\right)}
$$

for $\lambda \in \Lambda^{\prime}$. Using (31) and the relation (30)(b) in $L\left(A^{\prime}\right)$ and $L(A)$, we see that

$$
\varphi_{i j}(x y)=\varphi_{i k}(x) \varphi_{k j}(y)
$$

for $x, y \in A^{\prime}$ and $i, j, k \neq$.

Now let $u_{i j}=\varphi_{i j}\left(1^{\prime}\right)$ for $i \neq j$, where $1^{\prime}$ is the identity in $A^{\prime}$. Then, taking $y=1^{\prime}$ and separately $x=1^{\prime}$ in (33), we have $\varphi_{i j}(x)=\varphi_{i k}(x) u_{k j}$ and $\varphi_{i j}(y)=u_{i k} \varphi_{k j}(y)$ for $x, y \in A^{\prime}$ and $i, j, k \neq$. So

$$
\varphi_{i j}(x y)=\varphi_{i k}(x) \varphi_{k j}(y)=\left(\varphi_{i j}(x) u_{j k}\right)\left(u_{k i} \varphi_{i j}(y)\right)=\varphi_{i j}(x) \cdot u_{j k}, u_{k i} \varphi_{i j}(y)
$$

for $x, y \in A^{\prime}$ and $i, j, k \neq$. Also, by $(32), u_{12} \in A^{s\left(\alpha_{1}\right)}$ and $u_{23} \in A^{s\left(\alpha_{2}\right)}$. So replacing $u_{1}$ and $u_{2}$ by scalar multiples, we may assume that $u_{1}=u_{12}$ and $u_{2}=u_{23}$. Thus, $\varphi_{31}$ is an algebra isomorphism of $A^{\prime}$ onto $A^{\left(u_{1}, u_{2}\right)}$. Moreover, for $\lambda \in \Lambda^{\prime}$,

$$
\varphi_{31}\left(\left(A^{\prime}\right)^{\lambda}\right)=A^{\varphi_{e}(\lambda)+s\left(\varepsilon_{3}-\varepsilon_{1}\right)}=A^{\varphi_{e}(\lambda)-s\left(\alpha_{1}\right)-s\left(\alpha_{2}\right)}=\left(A^{\left(u_{1}, u_{2}\right)}\right)^{\varphi_{e}(\lambda)} .
$$

So we have our conclusion with $\eta=\varphi_{31}$.

Proposition 9.7. Suppose $A$ is an alternative $\Lambda$-torus and $s \in \operatorname{Hom}(Q, \Lambda)$. Choose $0 \neq u_{i} \in A^{s\left(\alpha_{i}\right)}$ for $i=1,2$. Then, $L(A)^{(s)} \simeq_{Q \times \Lambda} L\left(A^{\left(u_{1}, u_{2}\right)}\right)$.

Proof. This follows from Lemma 9.6 as in §8. (See the proof of Proposition 8.4.)

We could introduce isotopy for alternative tori in the obvious fashion (as in Definition 8.3 in the Jordan case). However, in view of the following result, this will not be necessary.

Proposition 9.8. Suppose that $A$ is an alternative $\Lambda$-torus and $u_{1}, u_{2}$ are nonzero homogeneous elements of $A$. Then $A^{\left(u_{1}, u_{2}\right)} \simeq_{i g} A$.

Proof. Let $A^{\prime}=A^{\left(u_{1}, u_{2}\right)}$. Suppose first that $A$ is associative. Then, one checks that the map $x \mapsto\left(u_{1} u_{2}\right)^{-1} x$ is a graded algebra isomorphism of $A$ onto $A^{\prime}$. So we can assume that $A$ is not associative. Thus, $A^{\prime}$ is also not associative [Mc1, p.256-257]. Therefore, by Theorem 9.3, $A$ and $A^{\prime}$ are both isograded-isomorphic to the octonion $\mathbb{Z}^{n}$-torus. So $A \simeq_{i g} A^{\prime}$.

Remark 9.9. With the assumptions of Proposition 9.8 one can actually show that $A^{\left(u_{1}, u_{2}\right)} \simeq_{\Lambda} A$, but isograded isomorphism is all that we need.

If $A$ is an alternative $\Lambda$-torus, then the opposite torus of $A$ is the alternative $\Lambda$-torus $A^{\mathrm{op}}$, where $A^{\mathrm{op}}=A$ as graded vector spaces and the product on $A^{\mathrm{op}}$ is given by $x \cdot$ op $y=y x$.

Lemma 9.10. Suppose that $A$ is an alternative $\Lambda$-torus. Then, there exists a unique bi-isomorphism from $L(A)$ onto $L\left(A^{o p}\right)$ such that

$$
e_{i j}(x) \mapsto-\tilde{e}_{j i}(x)
$$

for $x \in A$ and $i \neq j$, where $L\left(A^{o p}\right)=\tilde{L}_{0} \oplus\left(\bigoplus_{1 \leq i \neq j \leq 3} \tilde{e}_{i j}\left(A^{o p}\right)\right)$ as in $(29)$. 
Proof. In $L\left(A^{\mathrm{op}}\right)$, put $e_{i j}^{\prime}(x)=-\tilde{e}_{j i}(x)$ for $x \in A, i \neq j$, and put $L_{0}^{\prime}=\tilde{L}_{0}$. Then, $L\left(A^{\mathrm{op}}\right)=L_{0}^{\prime} \oplus\left(\bigoplus_{1 \leq i \neq j \leq 3} e_{i j}^{\prime}\left(A^{\mathrm{op}}\right)\right)$, and for $x, y \in A$ and $i, j, k \neq$, we have

$$
\left[e_{i j}^{\prime}(x), e_{j k}^{\prime}(y)\right]=\left[\tilde{e}_{j i}(x), \tilde{e}_{k j}(y)\right]=-\left[\tilde{e}_{k j}(y), \tilde{e}_{j i}(x)\right]=-\tilde{e}_{k i}(y \cdot \text { op } x)=e_{i k}^{\prime}(x y) .
$$

The other properties in (30) are clear, so we have a unique Lie algebra isomorphism $\varphi: L(A) \rightarrow L\left(A^{\mathrm{op}}\right)$ such that $\varphi\left(e_{i j}(x)\right)=e_{i j}^{\prime}(x)=-\tilde{e}_{j i}(x)$ for $x \in A$ and $i \neq j$. It is clear that $\varphi$ is a bi-isomorphism with $\varphi_{r}=-1$ and $\varphi_{e}=1$.

The main result of this section is:

Theorem 9.11. Suppose that $A$ is an alternative $\Lambda$-torus and $A^{\prime}$ is an alternative $\Lambda^{\prime}$-torus. Let $L(A)=\mathrm{pe}_{3}(A)$ and $L\left(A^{\prime}\right)=\mathrm{pe}_{3}\left(A^{\prime}\right)$. Then, the following are equivalent: ${ }^{13}$

(a) $A$ is isograded-isomorphic to $A^{\prime}$ or $\left(A^{\prime}\right)^{o p}$.

(b) $L(A) \simeq_{b i} L\left(A^{\prime}\right)$.

(c) $L(A) \sim L\left(A^{\prime}\right)$.

Proof. " $(\mathrm{a}) \Rightarrow(\mathrm{b})$ " Since $L\left(A^{\prime}\right) \simeq_{b i} L\left(A^{\prime \text { op }}\right)$ by Lemma 9.10, we can assume that $A \simeq_{i g} A^{\prime} .(\mathrm{b})$ is then clear.

"(b) $\Rightarrow$ (c)" is trivial.

"(c) $\Rightarrow(\mathrm{a})$ " By assumption, $L\left(A^{\prime}\right) \simeq_{b i} L(A)^{(s)}$ for some $s \in \operatorname{Hom}(Q, \Lambda)$ that is admissible for $L(A)$. Choose $0 \neq u_{i} \in A^{s\left(\alpha_{i}\right)}$ for $i=1,2$. Then, by Propositions 9.7 and 9.8 , we have $L(A)^{(s)} \simeq_{b i} L\left(A^{\left(u_{1}, u_{2}\right)}\right) \simeq_{b i} L(A)$. So there is a bi-isomorphism $\varphi: L\left(A^{\prime}\right) \rightarrow L(A)$. Now the automorphism group of $\Delta$ is generated by -1 and the Weyl group of $\Delta$. Hence, post-multiplying $\varphi$ by the bi-isomorphism in Lemma 9.10 if necessary, we can assume that $\varphi_{r}$ is in the Weyl group. Thus, by Lemma 3.8, we can assume that $\varphi_{r}=1$. So, by Lemma 9.6 (with $s=0$ and $u_{1}=u_{2}=1$ ), we have $A^{\prime} \simeq_{i g} A$.

Remark 9.12. If $A$ is an alternative torus, it is not in general true that $A$ is isogradedisomorphic to $A^{\text {op }}$. However, one can show that this is true if $A$ is the octonion torus (since the octonion torus has an involution) and if $A$ is a rational quantum torus (using the classification in $[\mathrm{Ne}]$ ).

\section{Type $\mathrm{A}_{r}, r \geq 3$}

Suppose in this section that $r \geq 3$,

$$
\Delta=\{0\} \cup\left\{\varepsilon_{i}-\varepsilon_{j} \mid 1 \leq i \neq j \leq r+1\right\}
$$

is the root system of type $\mathrm{A}_{r}$ (where $\varepsilon_{1}, \ldots, \varepsilon_{r}$ is a basis for a space containing $\Delta$ ), and $Q=Q(\Delta)$. We let $\alpha_{i}=\varepsilon_{i}-\varepsilon_{i+1}$ for $1 \leq i \leq r$, in which case $\left\{\alpha_{1}, \ldots, \alpha_{r}\right\}$ is a base for the root system $\Delta$.

Let $A$ be an associative $\Lambda$-torus, and denote by $[A, A]$ the Lie algebra spanned by commutators in $A$. Let

${ }_{13}$ See [GN, Lemma 1.4] for a result related to Theorems 9.11 and 10.6 in the context of Jordan pairs covered by grids. 


$$
L(A)=\mathrm{sl}_{r+1}(A):=\left\{X \in \operatorname{Mat}_{r+1}(A) \mid \operatorname{tr}(X) \in[A, A]\right\} .
$$

Then $L(A)$ is a Lie algebra under the commutator product called the $(r+1) \times(r+1)$ special linear Lie algebra over $A$. For $1 \leq i, j \leq r+1$, we let $e_{i j}$ be the $(i, j)$-matrix unit in $\operatorname{Mat}_{r+1}(A)$ with 1 in the $(i, j)$-position and zeros elsewhere, and we let $e_{i j}(x)=x e_{i j}$ for $x \in A$. Then $L(A)$ is a $Q \times \Lambda$-graded algebra with

$$
L(A)_{\varepsilon_{i}-\varepsilon_{j}}^{\lambda}=e_{i j}\left(A^{\lambda}\right), \quad L(A)_{0}^{\lambda}=\left\{\sum_{i=1}^{r+1} e_{i i}\left(x_{i}\right) \mid x_{i} \in A^{\lambda}, \sum_{i=1}^{r+1} x_{i} \in[A, A]\right\}
$$

for $i \neq j, \lambda \in \Lambda$, and $L(A)_{\alpha}^{\lambda}=0$ for $\lambda \in \Lambda, \alpha \in Q \backslash \Delta$. It is easy to check that the matrices $e_{i j}(x), x \in A, i \neq j$, generate the Lie algebra $L(A)$ and also, using the fact that the centre of $A$ intersects trivially with $[A, A]$ [BGK, Prop. 2.44], that $L(A)$ is centreless. ${ }^{14}$ It then follows easily that $L(A)$ is a centreless Lie $\Lambda$-torus of type $\Delta$.

Moreover, Berman, Gao and Krylyuk (over $\mathbb{C}$ ) and Yoshii (in general) proved the following coordinatization theorem:

Theorem 10.1. ([BGK, Thm. 2.65], [Y2, §4]) Any centreless Lie $\Lambda$-torus of type $\Delta$ is graded-isomorphic to the Lie $\Lambda$-torus $L(A)=\operatorname{sl}_{r+1}(A)$ constructed from an associative $\Lambda$-torus $A$.

Remark 10.2. Exactly as above one can define centreless Lie tori $\operatorname{sl}_{2}(A)$ and $\operatorname{sl}_{3}(A)$ in rank 1 and 2 respectively. Then it is not difficult to show that $\operatorname{sl}_{2}(A) \simeq_{Q \times A}$ $\operatorname{TKK}\left(A^{+}\right)$and $\operatorname{sl}_{3}(A) \simeq_{Q \times \Lambda} \mathrm{pe}_{3}(A)$. However, as we've seen, these constructions do not provide all examples of Lie tori of type $\mathrm{A}_{1}$ or $\mathrm{A}_{2}$.

To understand isotopes of Lie tori of type $\Delta$, we could proceed as in rank 1 and 2 to relate them to isotopes of the coordinate tori. However, we can take a simpler approach in view of the next proposition. ${ }^{15}$

Proposition 10.3. Suppose $A$ is an associative $\Lambda$-torus and $s \in \operatorname{Hom}(Q, \Lambda)$. (Note that as in rank 2 any $s \in \operatorname{Hom}(Q, \Lambda)$ is admissible for $L(A)$.) Then, $L(A)^{(s)} \simeq_{Q \times \Lambda}$ $L(A)$.

Proof. Let $Q^{\prime}=\bigoplus_{i=1}^{r+1} \mathbb{Z} \varepsilon_{i}$. Then $Q^{\prime}=\mathbb{Z} \varepsilon_{1} \oplus Q$, so we can extend $s$ to a group homomorphism $s: Q^{\prime} \rightarrow \Lambda$ by setting $s\left(\varepsilon_{1}\right)=0$. Choose $0 \neq d_{i} \in A^{s\left(\varepsilon_{i}\right)}$ for $1 \leq i \leq r+1$. Let $d=\operatorname{diag}\left(d_{1}, \ldots, d_{r+1}\right)$ and define $\varphi: L(A) \rightarrow L(A)$ by

$$
\varphi(X)=d X d^{-1}
$$

for $X \in L(A)$. (Here we are using the fact that $\operatorname{tr}\left(X_{1} X_{2}\right) \equiv \operatorname{tr}\left(X_{2} X_{1}\right)(\bmod [A, A])$ for $X_{1}, X_{2} \in \operatorname{Mat}_{r+1}(A)$, so $\varphi$ maps $L(A)$ into $L(A)$.) Then, $\varphi$ is a Lie algebra isomorphism. Also

$$
\begin{aligned}
\varphi\left(L(A)_{\varepsilon_{i}-\varepsilon_{j}}^{\lambda}\right) & =\varphi\left(e_{i j}\left(L^{\lambda}\right)\right)=e_{i j}\left(d_{i} A^{\lambda} d_{j}^{-1}\right)=e_{i j}\left(A^{\lambda+s\left(\varepsilon_{i}\right)-s\left(\varepsilon_{j}\right)}\right) \\
& =L(A)_{\varepsilon_{i}-\varepsilon_{j}}^{\lambda+s\left(\varepsilon_{i}-\varepsilon_{j}\right)}=\left(L(A)^{(s)}\right)_{\varepsilon_{i}-\varepsilon_{j}}^{\lambda}
\end{aligned}
$$

for $\lambda \in \Lambda$; so, by (LT3), $\varphi\left(L(A)_{\alpha}^{\lambda}\right)=\left(L(A)^{(s)}\right)_{\alpha}^{\lambda}$ for $\alpha \in Q, \lambda \in \Lambda$.

${ }^{14}$ In view of these properties, it is natural to also use the notation $\operatorname{pe}_{r+1}(A)$ for $L(A)$, which is consistent with the notation in rank 2 (see Remark 10.2).

15 This proposition could also be proved at the level of coordinates using the fact that any isotope of an associative algebra $A$ is isomorphic to $A$. 
The "key lemma" that we need is simpler than in rank 1 and 2 because of Proposition 10.3 .

Lemma 10.4 (Key lemma for type $\mathbf{A}_{r}, r \geq 3$ ). Suppose that $A^{\prime}$ is an associative $\Lambda^{\prime}$-torus and $A$ is an associative $\Lambda$-torus. Suppose that $\varphi: L\left(A^{\prime}\right) \rightarrow L(A)$ is a biisomorphism with $\varphi_{r}=1$. Then, there is an isograded-isomorphism $\eta: A^{\prime} \rightarrow A$ such that $\eta_{g r}=\varphi_{e}$.

Proof. By Lemma 3.8, we can assume that $\varphi\left(e_{i j}\left(1^{\prime}\right)\right)=e_{i j}(1)$ for $i-j= \pm 1$, and hence for all $i \neq j$. Also, we have the relation

$$
\left[e_{i j}(x), e_{j k}(y)\right]=e_{i k}(x y)
$$

in $L(A)$ for $x, y \in A$ and $i, j, k \neq$. We can thus argue as in the proof of Lemma 9.6 (with $s=0$ and $u_{1}=u_{2}=1$ ).

The following is easily checked:

Lemma 10.5. Suppose that $A$ is an associative $\Lambda$-torus. Then, the map $T \mapsto-T^{t}$ is a bi-isomorphism from $L(A)$ onto $L\left(A^{o p}\right)$.

Theorem 10.6. Suppose that $A$ is an associative $\Lambda$-torus and $A^{\prime}$ is an associative $\Lambda^{\prime}$-torus. Let $L(A)=\mathrm{sl}_{r+1}(A)$ and $L\left(A^{\prime}\right)=\mathrm{sl}_{r+1}\left(A^{\prime}\right)$. Then, the following are equivalent:

(a) $A$ is isograded-isomorphic to $A^{\prime}$ or $\left(A^{\prime}\right)^{o p}$.

(b) $L(A) \simeq_{b i} L\left(A^{\prime}\right)$.

(c) $L(A) \sim L\left(A^{\prime}\right)$.

Proof. If (a) holds then, by Lemma 10.5, we can assume that $A \simeq_{i g} A^{\prime}$, so (b) follows. The implication "(b) $\Rightarrow(\mathrm{c})$ " is trivial. Finally, suppose that (c) holds. Thus, $L(A)^{(s)} \simeq_{b i} L\left(A^{\prime}\right)$ for some $s \in \operatorname{Hom}(Q, \Lambda)$, so $L(A) \simeq_{b i} L\left(A^{\prime}\right)$ by Proposition 10.3 . We can now obtain (a) using the argument in the proof of Theorem 9.11 (and using Lemmas 10.4 and 10.5).

\section{Type $\mathrm{C}_{r}, r \geq 4$}

Suppose in this section that $r \geq 4$,

$$
\Delta=\{0\} \cup\left\{\varepsilon_{i}-\varepsilon_{j} \mid 1 \leq i \neq j \leq r\right\}\left\{ \pm\left(\varepsilon_{i}+\varepsilon_{j}\right) \mid 1 \leq i \leq j \leq r\right\}
$$

is the root system of type $\mathrm{C}_{r}$ (where $\varepsilon_{1}, \ldots, \varepsilon_{r}$ is a basis for the space spanned by $\Delta)$, and $Q=Q(\Delta)$. We let $\alpha_{i}=\varepsilon_{i}-\varepsilon_{i+1}, 1 \leq i \leq r-1$, and $\alpha_{r}=2 \varepsilon_{r}$, in which case $\left\{\alpha_{1}, \ldots, \alpha_{r}\right\}$ is a base for the root system $\Delta$.

Let $(A, \iota)$ be an associative $\Lambda$-torus with involution. For convenience, write $\bar{x}=$ $\iota(x)$ for $x \in A$ and $\bar{X}=\left(\bar{x}_{i j}\right)$ for $X=\left(x_{i j}\right) \in \operatorname{Mat}_{r}(A)$. We write $A_{ \pm}=A_{ \pm, \iota}:=$ $\{x \in A \mid \bar{x}= \pm x\}$, in which case $A=A_{+} \oplus A_{-}$. Let

$$
\begin{aligned}
L(A, \iota)=\operatorname{ssp}_{2 r}(A, \iota):=\left\{\left[\begin{array}{cc}
X & Y \\
Z & -\bar{X}^{t}
\end{array}\right] \mid X, Y, Z \in \operatorname{Mat}_{r}(A),\right. \\
\left.\bar{Y}^{t}=Y, \bar{Z}^{t}=Z, \operatorname{tr}(X) \in A_{+}+[A, A]\right\} .
\end{aligned}
$$


Then $L(A, \iota)$ is a Lie algebra under the commutator product called the $(2 r) \times(2 r)$ special symplectic Lie algebra over $A$. To define a grading on $L(A, \iota)$, let

$$
\begin{gathered}
\ell_{i j}(x)=x e_{i, j}-\bar{x} e_{j+r, i+r} \quad \text { if } 1 \leq i \neq j \leq r, \quad \text { and } \\
m_{i j}(x)=x e_{i, j+r}+\bar{x} e_{j, i+r}, \quad n_{i j}(x)=x e_{i+r, j}+\bar{x} e_{j+r, i} \quad \text { if } 1 \leq i, j \leq r,
\end{gathered}
$$

where $e_{i j}$ is the $(i, j)$-matrix unit. With this notation, $L(A, \iota)$ is a $Q \times \Lambda$-graded algebra with

$$
\begin{gathered}
L(A, \iota)_{\varepsilon_{i}-\varepsilon_{j}}^{\lambda}=\ell_{i j}\left(A^{\lambda}\right) \quad \text { for } 1 \leq i \neq j \leq r, \\
L(A, \iota)_{\varepsilon_{i}+\varepsilon_{j}}^{\lambda}=m_{i j}\left(A^{\lambda}\right) \quad \text { for } 1 \leq i, j \leq r, \\
L(A, \iota)_{-\left(\varepsilon_{i}+\varepsilon_{j}\right)}^{\lambda}=n_{i j}\left(A^{\lambda}\right) \quad \text { for } 1 \leq i, j \leq r,
\end{gathered}
$$

$$
L(A, \iota)_{0}^{\lambda}=\left\{\sum_{i=1}^{r}\left(e_{i, i}\left(x_{i}\right)-e_{i+r, i+r}\left(\bar{x}_{i}\right)\right) \mid x_{i} \in A^{\lambda}, \sum_{i=1}^{r} x_{i} \in A_{+}+[A, A]\right\}
$$

for $\lambda \in \Lambda$, and $L(A, \iota)_{\alpha}^{\lambda}=0$ for $\lambda \in \Lambda, \alpha \in Q \backslash \Delta$. Then $L(A, \iota)$ is a centreless Lie $\Lambda$-torus of type $\Delta$.

Moreover, Allison and Gao (over $\mathbb{C}$ ) and Benkart and Yoshii (in general) proved:

Theorem 11.1. ([AG, Prop. 4.87], [BY, Theorem 5.9]) Any centreless Lie A-torus of type $\Delta$ is graded-isomorphic to the Lie $\Lambda$-torus $L(A, \iota)=\operatorname{ssp}_{2 r}(A, \iota)$ constructed from an associative $\Lambda$-torus with involution $(A, \iota) .{ }^{16}$

Remark 11.2. Exactly as above one can define centreless Lie tori $\operatorname{ssp}_{4}(A, \iota)$ and $\operatorname{ssp}_{6}(A, \iota)$. However, these constructions do not provide all examples of Lie tori of type $\mathrm{C}_{2}=\mathrm{B}_{2}$ or $\mathrm{C}_{3}$ [AG, Thm. 4.87].

Example 11.3. Let $\mathbf{q}=\left(q_{i j}\right) \in \operatorname{Mat}_{n}(\mathbb{F})$ with $q_{i i}=1$ and $q_{i j}=q_{j i}= \pm 1$ for all $i, j$, and let $\mathbb{F}_{\mathbf{q}}=\mathbb{F}_{\mathbf{q}}\left[x_{1}^{ \pm 1}, \ldots, x_{n}^{ \pm 1}\right]$ be the quantum torus corresponding to $\mathbf{q}$ as in Example 7.3. Suppose $\mathbf{e}=\left(e_{1}, \ldots, e_{n}\right) \in \mathbb{F}^{n}$ with $e_{i}= \pm 1$, Then, there is a unique involution $\iota_{\mathbf{e}}$ on $\mathbb{F}_{\mathbf{q}}$ such that $\iota_{\mathbf{e}}\left(x_{i}\right)=e_{i} x_{i}$ for $1 \leq i \leq n$; and $\left(\mathbb{F}_{\mathbf{q}}, \iota_{\mathbf{e}}\right)$ is an associative $\mathbb{Z}^{n}$-torus with involution. ${ }^{17}$ It is not difficult to show that any associative torus with involution $(A, \iota)$ is isograded-isomorphic to some $\left(\mathbb{F}_{\mathbf{q}}, \iota_{\mathbf{e}}\right)$. More specifically, if we choose any basis $\left\{\lambda_{1}, \ldots, \lambda_{n}\right\}$ for $\Lambda$ and choose $0 \neq y_{i} \in A^{\lambda_{i}}$ for each $i$, then there is an isograded-isomorphism of $(A, \iota)$ onto $\left(\mathbb{F}_{\mathbf{q}}, \iota_{\mathbf{e}}\right)$ so that $y_{i} \mapsto x_{i}$ for each $i$, where $\mathbf{q}$ and $\mathbf{e}$ are determined by the equalities $y_{j} y_{i}=q_{i j} y_{i} y_{j}$ and $\iota\left(y_{i}\right)=e_{i} y_{i}$ [AG, p.163-164].

Yoshii in [Y3, Thm. 2.7] classified the associative tori with involution $\left(\mathbb{F}_{\mathbf{q}}, \iota_{\mathbf{e}}\right)$ up to isograded-isomorphism using successive changes of variables that transform the pair (q,e) into a canonical form. Alternatively, as we now see, one can classify associative tori with involution using quadratic forms following an approach discussed in $[\mathrm{AFY}$, Remark 5.20].

Let $\bar{\Lambda}=\Lambda / 2 \Lambda$ regarded as a vector space over $\mathbb{Z}_{2}=\mathbb{Z} / 2 \mathbb{Z}$, and let $\lambda \rightarrow \bar{\lambda}$ be the canonical map of $\Lambda$ onto $\bar{\Lambda}$. Recall that a map $\kappa: \bar{\Lambda} \rightarrow \mathbb{Z}_{2}$ is called a quadratic form

${ }^{16} L(A, \iota)$ was described differently in [AG] and also in [BY]. It is not difficult to check that the three descriptions give Lie tori that are $Q \times \Lambda$-graded-isomorphic.

${ }^{17}$ If $n=0$, we interpret $\left(\mathbb{F}_{\mathbf{q}}, \iota_{\mathbf{e}}\right)$ as $(\mathbb{F}$, id $)$. 
on $\bar{\Lambda}$ if the map $\kappa_{p}: \bar{\Lambda} \times \bar{\Lambda} \rightarrow \mathbb{Z}_{2}$ defined by $\kappa_{p}(\bar{\lambda}, \bar{\mu})=\kappa(\bar{\lambda}+\bar{\mu})+\kappa(\bar{\lambda})+\kappa(\bar{\mu})$ is biadditive. In that case, $\kappa_{p}$ is called the polarization of $\kappa$. (The usual assumptions that $\kappa(a \bar{\lambda})=a^{2} \kappa(\bar{\lambda})$ for $a \in \mathbb{Z}_{2}$ and that $\kappa_{p}$ is $\mathbb{Z}_{2}$-bilinear are automatic.) If $\Lambda$ has basis $\left\{\lambda_{1}, \ldots, \lambda_{n}\right\}$, then the quadratic forms on $\bar{\Lambda}$ are precisely the maps $\kappa: \bar{\Lambda} \rightarrow \mathbb{Z}_{2}$ of the form

$$
\kappa\left(\sum_{i=1}^{n} l_{i} \bar{\lambda}_{i}\right)=\sum_{i=1}^{n} l_{i} b_{i}+\sum_{1 \leq i \leq j \leq n} l_{i} l_{j} a_{i j},
$$

where $\left(b_{i}\right) \in \mathbb{Z}_{2}^{n}$ and $\left(a_{i j}\right)$ is an alternating matrix in $\operatorname{Mat}_{n}\left(\mathbb{Z}_{2}\right)$ (that is $a_{i j}=a_{j i}$ and $\left.a_{i i}=0\right)$.

Proposition 11.4. (i) If $(A, \iota)$ is an associative $\Lambda$-torus with involution, then there is a unique quadratic form $\kappa: \bar{\Lambda} \rightarrow \mathbb{Z}_{2}$, which we call the mod-2 quadratic form for $(A, \iota)$, such that

$$
\iota\left(x_{\lambda}\right)=(-1)^{\kappa(\bar{\lambda})} x_{\lambda}
$$

for $x_{\lambda} \in A^{\lambda}, \lambda \in \Lambda$. In that case, we also have

$$
x_{\lambda} x_{\mu}=(-1)^{\kappa_{p}(\bar{\lambda}, \bar{\mu})} x_{\mu} x_{\lambda}
$$

for $x_{\lambda} \in A^{\lambda}, x_{\mu} \in A^{\mu}, \lambda, \mu \in \Lambda$.

(ii) Any quadratic form $\kappa$ on $\bar{\Lambda}$ is the mod-2 quadratic form for some associative $\Lambda$-torus with involution. In fact, if $\Lambda=\mathbb{Z}^{n}$ with standard basis $\left\{\lambda_{1}, \ldots, \lambda_{n}\right\}, \kappa$ is given by (34) and we define $\mathbf{e}$ and $\mathbf{q}$ by $e_{i}=(-1)^{b_{i}}$ and $q_{i j}=(-1)^{a_{i j}}$, then $\kappa$ is the mod-2 quadratic form for $\left(\mathbb{F}_{\mathbf{q}}, \iota_{\mathbf{e}}\right)$.

(iii) If $(A, \iota)$ is an associative $\Lambda$-torus with involution and $\left(A^{\prime}, \iota^{\prime}\right)$ is an associative $\Lambda^{\prime}$-torus with involution, then $(A, \iota) \simeq_{i g}\left(A^{\prime}, \iota^{\prime}\right)$ if and only the corresponding mod-2 quadratic forms $\kappa$ and $\kappa^{\prime}$ are isometric, which means that there is an $\mathbb{Z}_{2}$-linear isomorphism $\bar{\tau}: \bar{\Lambda} \rightarrow \bar{\Lambda}^{\prime}$ such that $\kappa^{\prime}(\bar{\tau}(\bar{\lambda}))=\kappa(\bar{\lambda})$ for $\bar{\lambda} \in \bar{\Lambda}$.

Proof. We may assume that $\Lambda=\mathbb{Z}^{n}$ with standard basis $\left\{\lambda_{1}, \ldots, \lambda_{n}\right\}$, and in (iii) that $\Lambda^{\prime}=\mathbb{Z}^{n}$.

(i) The uniqueness of $\kappa$ is clear. For the existence we may assume that $(A, \iota)=$ $\left(\mathbb{F}_{\mathbf{q}}, \iota_{\mathbf{e}}\right)$ as in Example 11.3. Choose $\left(b_{i}\right) \in \mathbb{Z}_{2}^{n}$ and alternating $\left(a_{i j}\right) \in \operatorname{Mat}_{n}\left(\mathbb{Z}_{2}\right)$ such that $e_{i}=(-1)^{b_{i}}$ and $q_{i j}=(-1)^{a_{i j}}$. Then an easy computation shows that (35) holds with $\kappa$ given by (34). Also, if $x_{\lambda} \in A^{\lambda}, x_{\mu} \in A^{\mu}, \lambda, \mu \in \Lambda$, then $x_{\lambda} x_{\mu}=$ $\iota\left(\iota\left(x_{\mu}\right) \iota\left(x_{\lambda}\right)\right)=(-1)^{\kappa(\bar{\lambda}+\bar{\mu})+\kappa(\bar{\lambda})+\kappa(\bar{\mu})} x_{\mu} x_{\lambda}$, so (36) holds. of (i).

(ii) It is enough to prove the second statement which follows from the proof

(iii) We may assume that $(A, \iota)=\left(\mathbb{F}_{\mathbf{q}}, \iota_{\mathbf{e}}\right)$ as in Example 11.3. For " $\Rightarrow$ ", let $\eta:(A, \iota) \rightarrow\left(A^{\prime}, \iota^{\prime}\right)$ be an isograded-isomorphism and set $\tau:=\eta_{\mathrm{gr}} \in \mathrm{GL}(\Lambda)$. Then, $\eta\left(A^{\lambda}\right)=A^{\prime \tau(\lambda)}$ and so $\kappa^{\prime}(\overline{\tau(\lambda)})=\kappa(\bar{\lambda})$ for $\lambda \in \Lambda$. Thus, the map $\bar{\tau} \in \operatorname{GL}(\bar{\Lambda})$ induced by $\tau$ is an isometry of $\kappa$ onto $\kappa^{\prime}$. For " $\Leftarrow$ ", let $\bar{\tau} \in \mathrm{GL}(\bar{\Lambda})$ be an isometry of $\kappa$ onto $\kappa^{\prime}$. Then $\bar{\tau}$ is induced by some $\tau \in \operatorname{GL}(\Lambda)$. (This follows from the fact that any element of $\mathrm{GL}_{n}\left(\mathbb{Z}_{2}\right)$ is the product of elementary matrices.) Let $\lambda_{i}^{\prime}=\tau\left(\lambda_{i}\right)$ and $0 \neq x_{i}^{\prime} \in A^{\prime \lambda_{i}^{\prime}}$ for each $i$. Then, $x_{j}^{\prime} x_{i}^{\prime}=(-1)^{\kappa_{p}^{\prime}\left(\overline{\lambda_{i}^{\prime}}, \overline{\lambda_{j}^{\prime}}\right)} x_{i}^{\prime} x_{j}^{\prime}=(-1)^{\kappa_{p}\left(\bar{\lambda}_{i}, \bar{\lambda}_{j}\right)} x_{i}^{\prime} x_{j}^{\prime}=$ $q_{i j} x_{i}^{\prime} x_{j}^{\prime}$ and $\iota^{\prime}\left(x_{i}^{\prime}\right)=(-1)^{\kappa^{\prime}\left(\overline{\lambda_{i}^{\prime}}\right)} x_{i}^{\prime}=(-1)^{\kappa\left(\bar{\lambda}_{i}\right)} x_{i}^{\prime}=e_{i} x_{i}^{\prime}$, so $\left(A^{\prime}, \iota^{\prime}\right) \simeq_{i g}\left(\mathbb{F}_{\mathbf{q}}, \iota_{\mathbf{e}}\right)($ see Example 11.3). 
Finite dimensional quadratic forms over $\mathbb{Z}_{2}$ have been classified up to isometry [D, Chap. I, §16], and hence Proposition 11.4 gives a corresponding classification of associative $\Lambda$-tori with involution.

Remark 11.5. Suppose that $(A, \iota)$ is an associative $\Lambda$-torus with involution with $A_{+}=A_{+, \iota}$, and set $\Lambda_{+}=\Lambda_{+}(A, \iota):=\left\{\lambda \in \Lambda \mid A^{\lambda} \subseteq A_{+}\right\}$. If $1 \leq i \neq j \leq r$, then $\ell_{i j}(A), m_{i j}(A)$, and $n_{i j}(A)$ are subspaces of $L(A, \iota)$ and the maps $x \mapsto \ell_{i j}(x)$, $x \mapsto m_{i j}(x)$ and $x \mapsto n_{i j}(x)$ are injections from $A$ onto $\ell_{i j}(A), m_{i j}(A)$, and $n_{i j}(A)$ respectively. On the other hand if $1 \leq i \leq r$, we have $m_{i i}(x)=m_{i i}(\bar{x})$ and $n_{i i}(x)=n_{i i}(\bar{x})$ in $L(A, \iota)$ for $x \in A$, and the maps $h \mapsto m_{i i}(h)$ and $h \mapsto n_{i i}(h)$ are injections of $A_{+}$onto $m_{i i}\left(A_{+}\right)=m_{i i}(A)$ and $n_{i i}\left(A_{+}\right)=n_{i i}(A)$ respectively. Thus, if $\alpha \in \Delta^{\times}$, we have

$$
\Lambda_{\alpha}(L(A, \iota))= \begin{cases}\Lambda_{1} & \text { if } \alpha \text { is short; } \\ \Lambda_{+}, & \text {if } \alpha \text { is long. }\end{cases}
$$

Hence, if $s \in \operatorname{Hom}(Q, \Lambda)$, then $s$ is admissible for $L(A, \iota)$ if and only if $s\left(\alpha_{r}\right) \in \Lambda_{+}$.

If $(A, \iota)$ is an associative $\Lambda$-torus with involution, elements of $A_{+, \iota}$ are called hermitian elements of $(A, \iota)$. To form an isotope of $(A, \iota)$ along the lines of previous cases, we could a define new product $(x, y) \mapsto x h y$ on $A$, where $h$ is a nonzero homogeneous hermitian element of $(A, \iota)$, shift the grading, but keep the same involution. Following [Mc2, §I.3.4], an equivalent but simpler approach is to keep the product and grading and modify the involution.

Definition 11.6 (Isotopes of involutions). Suppose that $(A, \iota)$ is an associative $\Lambda$-torus with involution. Let $h$ be a nonzero homogeneous hermitian element of $(A, \iota)$, and define $\iota^{(h)}: A \rightarrow A$ by

$$
\iota^{(h)}(x)=h \bar{x} h^{-1}
$$

for $x \in A$. Then, $\left(A, \iota^{(h)}\right)$ is an associative $\Lambda$-torus with involution. (The multiplication and grading on $A$ are unchanged.) We call $\iota^{(h)}$ the $h$-isotope of $\iota$.

Lemma 11.7 (Key lemma for type $\mathbf{C}_{r}$ ). Suppose $\left(A^{\prime}, \iota^{\prime}\right)$ is an associative $\Lambda^{\prime}$ torus with involution, $(A, \iota)$ is an associative $\Lambda$-torus with involution, and $s \in$ $\operatorname{Hom}(Q, \Lambda)$ is admissible for $L(A, \iota)$. Suppose that $\varphi: L\left(A^{\prime}, \iota^{\prime}\right) \rightarrow L(A, \iota)^{(s)}$ is a bi-isomorphism with $\varphi_{r}=1$. Choose $0 \neq h \in A^{s\left(\alpha_{r}\right)}$. Then there is an isogradedisomorphism $\eta:\left(A^{\prime}, \iota^{\prime}\right) \rightarrow\left(A, \iota^{(h)}\right)$ such that $\eta_{g r}=\varphi_{e} \in \mathrm{GL}(\Lambda)$.

Proof. In the argument $i, j, k$ will always denote integers with $1 \leq i, j, k \leq r$.

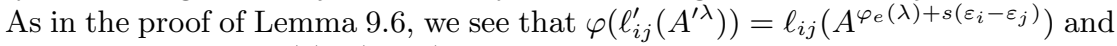
$\varphi\left(m_{i j}^{\prime}\left(A^{\prime \lambda}\right)\right)=m_{i j}\left(A^{\varphi_{e}(\lambda)+s\left(\varepsilon_{i}+\varepsilon_{j}\right)}\right)$ for $\lambda \in \Lambda$ and $i \neq j$. So we may define linear bijections $\varphi_{i j}: A^{\prime} \rightarrow A$ and $\psi_{i j}: A^{\prime} \rightarrow A$ for $i \neq j$ such that

$$
\varphi\left(\ell_{i j}(x)\right)=\ell_{i j}\left(\varphi_{i j}(x)\right), \quad \varphi\left(m_{i j}(x)\right)=m_{i j}\left(\psi_{i j}(x)\right)
$$

for $x \in A^{\prime}$, and

$$
\varphi_{i j}\left(A^{\prime \lambda}\right)=A^{\varphi_{e}(\lambda)+s\left(\varepsilon_{i}-\varepsilon_{j}\right)}, \quad \psi_{i j}\left(A^{\prime \lambda}\right)=A^{\varphi_{e}(\lambda)+s\left(\varepsilon_{i}+\varepsilon_{j}\right)}
$$

for $\lambda \in \Lambda$.

Next, direct multiplication in $L(A, \iota)$ gives the following identities: 


$$
\left[\ell_{i k}(x), \ell_{k j}(y)\right]=\ell_{i j}(x y) \quad \text { and } \quad\left[\ell_{i j}(x),\left[\ell_{j i}(y), m_{i j}(z)\right]\right]=m_{i j}(x(y z+\overline{y z}))
$$

for $x, y, z \in A, i, j, k \neq$. We have similar equations in $L\left(A^{\prime}, \iota^{\prime}\right)$, and applying $\varphi$ to these yields

$$
\begin{aligned}
\varphi_{i j}(x y) & =\varphi_{i k}(x) \varphi_{k j}(y) \\
\psi_{i j}(x(y z+\overline{y z})) & =\varphi_{i j}(x)\left(\varphi_{j i}(y) \psi_{i j}(z)+\overline{\varphi_{j i}(y) \psi_{i j}(z)}\right)
\end{aligned}
$$

for $x, y, z \in A^{\prime}, i, j, k \neq$.

We let $u_{i j}=\varphi_{i j}\left(1^{\prime}\right) \in A^{s\left(\varepsilon_{i}-\varepsilon_{j}\right)}$ and $v_{i j}=\psi_{i j}\left(1^{\prime}\right) \in A^{s\left(\varepsilon_{i}+\varepsilon_{j}\right)}$ for $i \neq j$. Setting $x=y=1^{\prime}$ in (37), we get $u_{i j}=u_{i k} u_{k j}$ for $i, j, k \neq$. Therefore, $u_{i j} u_{j i}=$ $u_{k i}^{-1} u_{k i} u_{i j} u_{j i}=u_{k i}^{-1} u_{k j} u_{j i}=u_{k i}^{-1} u_{k i}=1$, so $u_{i j}=u_{j i}^{-1}$ for $i \neq j$.

As in the proof of Lemma 9.6, it follows from (37) that we have $\varphi_{i j}(x y)=$ $\left(\varphi_{i j}(x) u_{j k}\right)\left(u_{k i} \varphi_{i j}(y)\right)$ for $x, y \in A^{\prime}, i, j, k \neq$. So $\varphi_{i j}(x y)=\varphi_{i j}(x) u_{j i} \varphi_{i j}(y)$ for $i \neq j$. Thus, we see that $\chi_{i j}: A^{\prime} \rightarrow A$, defined by

$$
\chi_{i j}(x)=u_{j i} \varphi_{i j}(x)
$$

is an algebra isomorphism of $A^{\prime}$ onto $A$ for $i \neq j$. Also, $\chi_{i j}\left(A^{\prime \lambda}\right)=u_{j i} \varphi_{j i}\left(A^{\prime \lambda}\right)=$ $u_{j i} A^{\varphi_{e}(\lambda)+s\left(\varepsilon_{i}-\varepsilon_{i}\right)} \subseteq A^{\varphi_{e}(\lambda)}$ for $\lambda \in \Lambda$, so $\chi_{i j}$ is isograded with $\left(\chi_{i j}\right)_{\mathrm{gr}}=\varphi_{e}$.

If we put $x=y=1^{\prime}$ in (38), we get $\psi_{i j}(z+\bar{z})=u_{i j}\left(u_{j i} \psi_{i j}(z)+\overline{u_{j i} \psi_{i j}(z)}\right)$. Since $u_{i j} u_{j i}=1$, this yields

$$
\psi_{i j}(\bar{z})=u_{i j} \overline{\psi_{i j}(z)} \bar{u}_{j i}
$$

for $z \in A^{\prime}, i \neq j$. Also, if we take $y=z=1^{\prime}$ in (38), we get

$$
\psi_{i j}(2 x)=\varphi_{i j}(x)\left(u_{j i} v_{i j}+\overline{u_{j i} v_{i j}}\right)
$$

for $x \in A^{\prime}, i \neq j$.

Next $u_{r 1} v_{1, r} \in A^{s\left(\varepsilon_{r}-\varepsilon_{1}\right)} A^{s\left(\varepsilon_{r}+\varepsilon_{1}\right)} \subseteq A^{s\left(2 \varepsilon_{r}\right)}$. So replacing $h$ by a scalar multiple, we can assume that $h=u_{r 1} v_{1, r}$. Since $s$ is admissible for $L(A, \iota)$, we have $\bar{h}=h$, so (41) tells us that

$$
\psi_{1 r}(x)=\varphi_{1 r}(x) h
$$

for $x \in A^{\prime}$. Finally, using (39), (40) and (42), we have

$$
\begin{aligned}
\chi_{1 r}(\bar{x}) & =u_{r 1} \varphi_{1 r}(\bar{x})=u_{r 1} \psi_{1 r}(\bar{x}) h^{-1}=u_{r 1} u_{1 r} \overline{\psi_{1 r}(x)} \bar{u}_{r 1} h^{-1} \\
& =\overline{\psi_{1 r}(x)} \bar{u}_{r 1} h^{-1}=h \overline{\varphi_{1 r}(x)} \bar{u}_{r 1} h^{-1}=h \overline{\chi_{1 r}(x)} h^{-1}
\end{aligned}
$$

for $x \in A^{\prime}$. Thus, $\chi_{1 r}$ is an isograded isomorphism of $\left(A^{\prime}, \iota^{\prime}\right)$ onto $\left(A, \iota^{(h)}\right)$.

As in types $A_{1}$ and $A_{2}$, the following proposition now follows from Theorem 11.1 and Lemma 11.7.

Proposition 11.8. Suppose that $(A, \iota)$ is associative $\Lambda$-torus with involution and $s \in \operatorname{Hom}(Q, \Lambda)$ is admissible for $L(A, \iota)$. Choose $0 \neq h \in A^{s\left(\alpha_{r}\right)}$. Then, we have $L(A, \iota)^{(s)} \simeq_{Q \times \Lambda} L\left(A, \iota^{(h)}\right)$.

We could follow the pattern in type $\mathrm{A}_{1}$ and define two associative tori with involution $(A, \iota)$ and $\left(A^{\prime}, \iota^{\prime}\right)$ to be isotopic if $\left(A, \iota^{(h)}\right)$ is isograded-isomorphic to $\left(A^{\prime}, \iota^{\prime}\right)$ for some nonzero homogeneous hermitian element $h$ of $(A, \iota)$. However, we don't need to do this because of the following: 
Proposition 11.9. Suppose that $(A, \iota)$ is an associative $\Lambda$-torus with involution and $h$ is a nonzero homogeneous hermitian element of $(A, \iota)$. Then $\left(A, \iota^{(h)}\right) \simeq_{i g}(A, \iota)$.

Proof. Let $\kappa$ and $\kappa^{(h)}$ be the mod-2 quadratic forms for $(A, \iota)$ and $\left(A, \iota^{(h)}\right)$ respectively. Now $h \in A^{\mu}$, where $\mu \in A$, and since $h$ is hermitian we have $\kappa(\bar{\mu})=0$. Also, if $x \in A^{\lambda}, \lambda \in A$, we have $\iota^{(h)}(x)=h \iota(x) h^{-1}=(-1)^{\kappa(\bar{\lambda})} h x h^{-1}=(-1)^{\kappa(\bar{\lambda})+\kappa_{p}(\bar{\lambda}, \bar{\mu})} x$, so $\kappa^{(h)}(\bar{\lambda})=\kappa(\bar{\lambda})+\kappa_{p}(\bar{\lambda}, \bar{\mu})$ for $\bar{\lambda} \in \bar{\Lambda}$. Hence, since $\kappa(\bar{\mu})=0$, we have

$$
\kappa^{(h)}(\bar{\lambda})=\kappa(\bar{\lambda})+\kappa_{p}(\bar{\lambda}, \bar{\mu})^{2}=\kappa\left(\bar{\lambda}+\kappa_{p}(\bar{\mu}, \bar{\lambda}) \bar{\mu}\right)
$$

for $\bar{\lambda} \in \bar{\Lambda}$. So we define $\bar{\tau}: \bar{\Lambda} \rightarrow \bar{\Lambda}$ by $\bar{\tau}(\bar{\lambda})=\bar{\lambda}+\kappa_{p}(\bar{\mu}, \bar{\lambda}) \bar{\mu}$ for $\bar{\lambda} \in \bar{\Lambda}$. One checks that $\bar{\tau}^{2}=1$, so $\bar{\tau}$ is an isometry of $\kappa^{(h)}$ onto $\kappa$. Hence, our conclusion follows from Proposition 11.4(iii).

Theorem 11.10. Suppose that $(A, \iota)$ is an associative $\Lambda$-torus with involution and $\left(A^{\prime}, \iota^{\prime}\right)$ is an associative $\Lambda^{\prime}$-torus with involution. Let $L(A, \iota)=\operatorname{ssp}_{2 r}(A, \iota)$ and $L\left(A^{\prime}, \iota^{\prime}\right)=\operatorname{ssp}_{2 r}\left(A^{\prime}, \iota^{\prime}\right)$. Then, the following are equivalent:

(a) $(A, \iota)$ is isograded-isomorphic to $\left(A^{\prime}, \iota^{\prime}\right)$.

(b) $L(A, \iota) \simeq_{b i} L\left(A^{\prime}, \iota^{\prime}\right)$.

(c) $L(A, \iota) \sim L\left(A^{\prime}, \iota^{\prime}\right)$.

Proof. " $(\mathrm{a}) \Rightarrow(\mathrm{b})$ " is clear, and " $(\mathrm{b}) \Rightarrow(\mathrm{c})$ " is trivial. Finally, suppose that (c) holds. Thus, $L\left(A^{\prime}, \iota^{\prime}\right) \simeq_{b i} L(A, \iota)$ by Propositions 11.8 and 11.9 , so there exists a biisomorphism $\varphi: L\left(A^{\prime}, \iota^{\prime}\right) \rightarrow L(A, \iota)$. Since the automorphism group of $\Delta$ equals the Weyl group of $\Delta$, we can, by Lemma 3.8, assume that $\varphi_{r}=1$. (a) now follows from Proposition 11.8 (with $s=0$ and $h=1$ ).

\section{Some concluding remarks}

Putting together the theorems quoted and proved in this article, we obtain a very good understanding of the structure of EALAs of several types.

Type $A_{1}$ : By Theorems 8.1 and Theorem 8.6, the TKK construction induces a 11 correspondence between isotopy classes of Jordan $\mathbb{Z}^{n}$-tori and isotopy classes of centreless Lie $\mathbb{Z}^{n}$-tori of type $A_{1}$. Composing this with Neher's EALA construction (Construction 4.1), we obtain, by the results of $\S 6$, an explicit 1-1 correspondence between isotopy classes of Jordan $\mathbb{Z}^{n}$-tori and families of EALAs of type $\mathrm{A}_{1}$ and nullity $n$ up to bijective isomorphism.

Type $A_{r}(r \geq 2)$ : By the results of $\S$ 's 6,9 and 10 , if $r=2$ (resp. $r \geq 3$ ) the $3 \times 3$ projective elementary construction (resp. the $(r+1) \times(r+1)$-special linear construction) followed by Neher's construction gives us an explicit 1-1 correspondence between isograded-isomorphism classes of alternative (resp. associative) $\mathbb{Z}^{n}$-tori and families of EALAs of type $\mathrm{A}_{r}$ and nullity $n$ up to bijective isomorphism.

Type $C_{r}(r \geq 4)$ : The structural information is particularly sharp in this case. Indeed, by Theorem 11.1 and Theorem 11.10 (again along with the results of $\S 6$ ), the construction of an associative torus with involution from a mod-2 quadratic form (see Proposition 11.4(ii)), followed by the $2 r \times 2 r$-special symplectic construction, 
followed by Neher's construction, gives an explicit 1-1 correspondence between isometry classes of quadratic forms on an $n$-dimensional vector space over $\mathbb{Z}_{2}$ and families of EALAs of type $\mathrm{C}_{r}$ and nullity $n$ up to bijective isomorphism.

Type $D_{r}(r \geq 4), E_{6}, E_{7}, E_{8}$ : Let $\mathrm{X}_{r}$ be one of the indicated types. According to [BGK, Thm. 1.37] and [Y2, §5], any centreless Lie $\Lambda$-torus of type $\mathrm{X}_{r}$ is isogradedisomorphic to the untwisted Lie torus $\mathfrak{g} \otimes \mathbb{F}[\Lambda]$, where $\mathfrak{g}$ is the split simple Lie algebra of type $\mathrm{X}_{r}$. Thus, there is only one bi-isomorphism class and hence only one isotopy class of centreless Lie $\mathbb{Z}^{n}$-tori of type $\mathrm{X}_{r}$. Therefore, for a given nullity, there is only one family of EALAs of type $\mathrm{X}_{r}$ up to bijective isomorphism.

Other types: We have omitted discussion of families of EALAs of types $\mathrm{C}_{3}, \mathrm{~B}_{r}(r \geq 2)$ and $\mathrm{BC}_{r}(r \geq 1)$. Type $\mathrm{C}_{3}$ can be handled using for the methods of $\S 11$, as there is only one centreless Lie $\mathbb{Z}^{n}$-torus of type $\mathrm{C}_{3}$ that is not covered by the $6 \times 6$ special symplectic construction ([AG, Thm. 4.87], [BY, Thm. 5.9]). (This exceptional example exists only if $n \geq 3$.) In types $\mathrm{B}_{r}(r \geq 2)$ and $\mathrm{BC}_{r}(r \geq 1)$, we expect that isotopes and isotopy of coordinate algebras will play a key role not only in the arguments but also in the statements of the results (as in type $\mathrm{A}_{1}$ ).

Finally, we note that when $\mathbb{F}$ is algebraically closed, there is an alternative method (replacing coordinatization) to construct centreless Lie $\mathbb{Z}^{n}$-tori. This method, which has been investigated recently in [ABFP1] and [ABFP2], constructs centreless Lie tori as multiloop algebras, just (as in nullity 1) affine Kac-Moody Lie algebras are constructed using loop algebras. To be somewhat more precise, suppose that $\boldsymbol{\sigma}=\left(\sigma_{1}, \ldots, \sigma_{n}\right)$ is an $n$-tuple of commuting finite order automorphisms of a finite dimensional simple Lie algebra $\mathfrak{s}$. Let $\mathfrak{g}=\mathfrak{s}^{\sigma}$ be the fixed point algebra of $\left\{\sigma_{i}\right\}_{i=1}^{n}$ in $\mathfrak{s}$, let $\mathfrak{h}$ be a Cartan subalgebra of $\mathfrak{g}$, let $\Delta=\Delta(\mathfrak{s}, \mathfrak{h})$, and let $Q=\operatorname{span}_{\mathbb{Z}} \Delta$. We give the tensor product $T=\mathfrak{s} \otimes \mathbb{F}\left[z_{1}^{ \pm 1}, \ldots, z_{n}^{ \pm 1}\right]$ a $Q \times \mathbb{Z}^{n}$-grading using the natural $Q$-grading of the first factor and the natural $\mathbb{Z}^{n}$-grading of the second factor. Next, for $1 \leq i \leq n$, we fix a primitive $m_{i}^{\text {th }}$ root of unity $\zeta_{m_{i}}$ for each $i$, where $m_{i}=\left|\sigma_{i}\right|$, and we define an automorphism $\eta_{i}$ of $\mathbb{F}\left[z_{1}^{ \pm 1}, \ldots, z_{n}^{ \pm 1}\right]$ by $\eta_{i}\left(z_{j}\right)=\zeta_{m_{i}}^{\delta_{i j}} z_{j}$. Now let $\operatorname{LT}(\mathfrak{s}, \boldsymbol{\sigma}, \mathfrak{h})$ be the fixed point algebra of $\left\{\sigma_{i} \otimes \eta_{i}^{-1}\right\}_{i=1}^{n}$ in $T$, in which case $\operatorname{LT}(\mathfrak{s}, \boldsymbol{\sigma}, \mathfrak{h})$ is a $Q \times \mathbb{Z}^{n}$-graded subalgebra of $T$. If $\boldsymbol{\sigma}$ satisfies some additional conditions that are listed in [ABFP2, Prop. 3.2.5] (the most important being that $\mathfrak{g}$ is simple), then $\Delta$ is a root system in $\mathfrak{h}^{*}$ and $\operatorname{LT}(\mathfrak{s}, \boldsymbol{\sigma}, \mathfrak{h})$ is a centreless Lie $\mathbb{Z}^{n}$-torus of type $\Delta$, which is called a multiloop Lie torus. Also it is shown in [ABFP2, Thm. 3.3.1] that a centreless Lie torus $L$ is bi-isomorphic to some multiloop Lie torus if and only if $L$ is a finitely generated as a module over its centroid. Furthermore, necessary and sufficient conditions are given there for two multiloop Lie tori to be bi-isomorphic or to be isotopic. The reader can consult [ABFP1] and [ABFP2] for details about these results.

The two approaches, using coordinatization and using multiloops, which have been used to construct and to determine isotopy of centreless Lie tori, each have significant complimentary advantages. It is our view that both are needed in order to gain a full understanding of families of EALAs.

\section{References}

[A] A. Albert, Non-associative algebras. I. Fundamental concepts and isotopy, Ann. of Math. 43 (1942), 685-707. 
[AABGP] B. Allison, S. Azam, S. Berman, Y. Gao and A. Pianzola, Extended affine Lie algebras and their root systems, Mem. Amer. Math. Soc. 126 (1997), no. $603, \mathrm{x}+122 \mathrm{pp}$.

[AB] B. Allison and G. Benkart, Unitary Lie algebras and Lie tori of type $B C_{r}$, $r \geq 3$, in preparation.

[ABFP1] B. Allison, S. Berman, J. Faulkner, A. Pianzola, Realization of gradedsimple algebras as loop algebras, to appear in Forum Mathematicum, arXiv:math/0511723v2 [math.RA].

[ABFP2] B. Allison, S. Berman, J. Faulkner and A. Pianzola, Multiloop realization of extended affine Lie algebras and Lie tori, to appear in Trans. Amer. Math. Soc, arXiv:0709.0975v2 [math.RA].

[ABGP] B. Allison, S. Berman, Y. Gao and A. Pianzola, A characterization of affine Kac-Moody Lie algebras, Comm. Math. Phys. 185 (1997), 671-688.

[AFY] B Allison, J. Faulkner and Y. Yoshii, Structurable tori, to appear in Comm. Algebra, arXiv:math/0702678v1 [math.RA].

[AG] B. Allison and Y. Gao, The root system and the core of an extended affine Lie algebra, Selecta Math. (N.S.) 7 (2001), 149-212.

[BN] G. Benkart and E. Neher, The centroid of extended affine and root graded Lie algebras, J. Pure Appl. Algebra 205 (2006), 117-145.

[BiN] Y. Billig and K.-H. Neeb, On the cohomology of vector fields on parallelizable manifold, preprint, arXiv:0705.3382v1 [math.RT].

[BY] S. Benkart and Y. Yoshii, Lie G-tori of symplectic type, Q. J. Math. 57 (2006), 425-448.

[BGK] S. Berman, Y. Gao and Y. Krylyuk, Quantum tori and the structure of elliptic quasi-simple Lie algebras, J. Funct. Anal. 135 (1996), 339-389.

[BGKN] S. Berman, Y. Gao, Y. Krylyuk and E. Neher, The alternative torus and the structure of elliptic quasi-simple Lie algebras of type $\mathrm{A}_{2}$, Trans. Amer. Math. Soc. 347 (1995), 4315-4363.

[B1] N. Bourbaki, Lie groups and Lie algebras. Chapters 1-3, Translated from the French. Reprint of the 1975 edition. Elements of Mathematics (Berlin). Springer-Verlag, Berlin, 1989.

[B2] N. Bourbaki, Lie groups and Lie algebras. Chapters 4-6, Translated from the French, Elements of Mathematics (Berlin), Springer-Verlag, Berlin, 2002.

[B3] N. Bourbaki, Lie groups and Lie algebras. Chapters 7-9, Translated from the French, Elements of Mathematics (Berlin), Springer-Verlag, Berlin, 2005.

[D] J. Dieudonné, La géométrie des groupes classiques, Troisième édition. Ergebnisse der Mathematik und ihrer Grenzgebiete, Band 5. SpringerVerlag, Berlin-New York, 1971.

[F1] J. Faulkner, Barbilian planes, Geom. Dedicata 30 (1989), 125-181.

[F2] J. Faulkner, Lie tori of type $\mathrm{BC}_{2}$ and structurable quasitori, to appear in Comm. Algebra.

[GN] E. Garcia and E. Neher, Semiprime, prime and simple Jordan superpairs covered by grids, J. Algebra 273 (2004), 1-32.

[H] J.T. Hartwig, Locally finite simple weight modules over twisted generalized Weyl algebras, J. Algebra 303 (2006), 42-76.

[HT] R. Høegh-Krohn and B. Torresani, Classification and construction of quasi-simple Lie algebras, J. Funct. Anal. 89 (1990), 106-136. 
[Mc1] K. McCrimmon, Homotopes of alternative algebras, Math. Ann. 191, 253262.

[Mc2] K. McCrimmon, A taste of Jordan algebras, Springer, New York, 2004.

[MRY] R. Moody, S.E. Rao and T. Yokonuma, Toroidal Lie algebras and vertex representations. Geom. Dedicata 35 (1990) 283-307.

[Ne] K.-H. Neeb, On a normal form of the rational quantum tori and their automorphism groups, preprint, arXiv:math/0511263v5 [math.RA].

[N1] E. Neher, Lie tori, C.R. Math. Acad. Sci. Soc. R. Can., 26 (2004), 84-89.

[N2] E. Neher, Extended affine Lie algebras, C.R. Math. Acad. Sci. Soc. R. Can., 26 (2004), 90-96.

[S] R.D. Schafer, An introduction to nonassociative algebras. Pure and Applied Mathematics, Vol. 22 Academic Press, New York-London 1966.

[Y1] Y. Yoshii, Coordinate algebras of extended affine Lie algebras of type $A_{1}$, J. Algebra 234 (2000), 128-168.

[Y2] Y. Yoshii, Root-graded Lie algebras with compatible grading, Comm. Algebra 29 (2001), 3365-3391.

[Y3] Y. Yoshii, Classification of quantum tori with involution, Canad. Math. Bull. 45 (2002), 711-731.

[Y4] Y. Yoshii, Classification of $\mathbb{Z}^{n}$-graded alternative algebras, J. Alg. 256 (2002), 28-50.

[Y5] Y. Yoshii, Root systems extended by an abelian group and their Lie algebras, J. Lie Theory 14 (2004), 371-394.

[Y6] Y. Yoshii, Lie tori-A simple characterization of extended affine Lie algebras, Publ. Res. Inst. Math. Sci. 42 (2006), 739-762.

[Y7] Y. Yoshii, Cayley polynomials, preprint, arXiv:math/0601570v1 [math.RA]. 